\title{
The Benefits of Autonomous Vehicles for Community-Based Trip Sharing
}

\author{
Mohd. Hafiz Hasan ${ }^{\mathrm{a}}$, Pascal Van Hentenryck ${ }^{\mathrm{b}, *}$ \\ ${ }^{a}$ University of Michigan, Ann Arbor, Michigan 48105, USA \\ ${ }^{b}$ Georgia Institute of Technology, Atlanta, Georgia 30332, USA
}

\begin{abstract}
This work reconsiders the concept of community-based trip sharing proposed by Hasan et al. (2018) that leverages the structure of commuting patterns and urban communities to optimize trip sharing. It aims at quantifying the benefits of autonomous vehicles for community-based trip sharing, compared to a car-pooling platform where vehicles are driven by their owners. In the considered problem, each rider specifies a desired arrival time for her inbound trip (commuting to work) and a departure time for her outbound trip (commuting back home). In addition, her commute time cannot deviate too much from the duration of a direct trip. Prior work motivated by reducing parking pressure and congestion in the city of Ann Arbor, Michigan, showed that a car-pooling platform for community-based trip sharing could reduce the number of vehicles by close to $60 \%$.

This paper studies the potential benefits of autonomous vehicles in further reducing the number of vehicles needed to serve all these commuting trips. It proposes a column-generation procedure that generates and assembles mini routes to serve inbound and outbound trips, using a lexicographic objective that first minimizes the required vehicle count and then the total travel distance. The optimization algorithm is evaluated on a large-scale, real-world dataset of commute trips from the city of Ann Arbor, Michigan. The results of the optimization show that it can leverage autonomous vehicles to reduce the daily vehicle usage by $92 \%$, improving upon the results of the original Commute Trip Sharing Problem by $34 \%$, while also reducing daily vehicle miles traveled by approximately $30 \%$. These results demonstrate the significant potential of autonomous vehicles for the shared commuting of a community to a common work destination.
\end{abstract}

Keywords: autonomous vehicles, shared commuting, vehicle routing problem, mixed-integer programming, column generation

\section{Introduction}

The notion of community-based trip sharing-leveraging the structure of commuting patterns and urban communities when optimizing trip sharing-was first explored by Hasan et al. (2018) to reduce parking pressure and congestion on university and corporate campuses. The

${ }^{*}$ Corresponding author

Email addresses: hasanm@umich.edu (Mohd. Hafiz Hasan), pascal . vanhentenryck@isye.gatech.edu (Pascal Van Hentenryck) 
study, which was originally motivated by the desire to relieve parking pressure at the University of Michigan, Ann Arbor, investigated the effects of different driver and commuter matching arrangements on trip shareability for a car-pooling or car-sharing platform. Trip shareability was loosely defined as the ability to aggregate as many trips as possible to reduce the number of vehicles required to serve them. The evaluation of several different optimization models revealed that commuter matching flexibility, i.e., a willingness to be matched with different drivers and passengers daily, is key for an effective trip-sharing platform.

This early work was extended by Hasan et al. (2020), where the Commute Trip Sharing Problem (CTSP) was formalized. The CTSP seeks a routing plan for the set of commuting trips that minimizes a lexicographic objective. The primary objective is to minimize the number of vehicles to cover all trips, while the secondary objective is to minimize the total travel distance. Every commuting trip consists of a pair of trip requests, one to the workplace (inbound) and another back home (outbound), each with specific pickup and drop-off locations as well as time windows specifying allowable service times at each location. The routes of the CTSP must serve each request exactly once and ensure that a vehicle capacity and trip specific ride-duration limits are not exceeded. The ride-duration constraint guarantees a level of quality of service for the riders. Finally, as the routing plan selects drivers from the set of commuters, it must ensure that the set of drivers selected for the inbound trips is identical to that for the outbound trips. The CTSP is thus a Vehicle Routing Problem (VRP) with time-window, capacity, pairing, precedence, ride-duration, and driver constraints. Hasan et al. (2020) proposed two exact algorithms to solve the CTSP and applied them on a real-world dataset from the city of Ann Arbor, Michigan. The case study shows that community-based car pooling can decrease daily vehicle usage by up to $57 \%$. These results highlighted the significant potential in vehicle reduction of community-based trip sharing. However, the vehicles in the CTSP routing plans are still mostly idle, as they perform a single inbound and outbound trip a day. Moreover, the constraint that a trip starts at the driver origin and ends at the driver destination limits the potential for ridesharing. These limitations make the adoption of autonomous vehicles (AVs) for the CTSP particularly appealing, as the absence of drivers would directly address these key shortcomings and could potentially lead to further reductions in fleet size, higher vehicle utilization, and increased ridesharing.

The goal of this paper is to examine the potential benefits of using AVs for performing the same trips, quantifying the reduction in fleet size and the miles travelled. It studies the Commute Trip Sharing Problem for Autonomous Vehicles (CTSPAV) which is similar to CTSP but uses a fleet of AVs that depart from and return to a designated depot to serve all commute trips. The CTSPAV is very similar to, and is a specialization of, the Dial-A-Ride Problem (DARP) (Cordeau, 2006). The main difference is that trip requests in the CTSPAV come in pairs, one to the workplace (typically in the morning) and another to return back home (typically in the evening). This feature makes it possible to adopt solution techniques that are computationally attractive. Our proposed solution approach involves chaining mini routes, i.e., short routes with distinct pickup, transit, and drop-off phases, to form longer AV routes. It is especially suited for problem scenarios involving commuters traveling to a common/centralized location, e.g., the commute trips of employees of a university or corporate campus, or those involving commuters living in a common/centralized location, e.g., the commute trips originating from an apartment complex or a residential neighborhood. The case study considered in this work, which consists of commute trips made to 15 university-owned parking structures located within close vicinity to each other in downtown Ann Arbor, Michigan, fits the profile of the former scenario, making it a prime candidate for evaluating the efficacy of the proposed approach.

The main contributions of this paper can be summarized as follows: 
1. The paper formalizes the CTSPAV that seeks an optimal set of routes for a fleet of AVs for serving a set of commute trips subject to the passenger-related constraints of the original CTSP.

2. The paper proposes a column-generation procedure to find a high-quality solution to the problem. The procedure uses a pricing problem to generate mini routes that are then assembled in a master problem. Each mini route serves inbound (resp. outbound) trips for a number of riders, satisfying time-window and ride-duration constraints on the trips as well as the capacity constraints on the vehicles. This approach is a departure from classical column-generation procedures commonly adopted for VRPs and the DARP, whereby the pricing problem searches for complete routes departing from and returning to a depot, while the master problem solves a set-covering/partitioning problem that ensures every customer is served.

3. The paper shows that the proposed algorithm outperforms a state-of-the-art DARP algorithm based on the classical column-generation approach for the CTSPAV.

4. The paper applies the proposed algorithms on a large-scale, real-world dataset of commute trips for the city of Ann Arbor, Michigan. The experimental results show that the algorithm is capable of reducing the daily vehicle usage by $92 \%$, improving upon the results of the original CTSP by $34 \%$, while also reducing daily vehicle miles traveled by approximately $30 \%$.

Overall, the results demonstrate the significant potential of AVs for serving the commuting needs of a community whose members work at a common location. The paper also includes a coarse cost analysis that highlights that fleet sizing is the correct metric to optimize when the goal consists of maximizing the profitability of the service.

The rest of this paper is organized as follows. Section 2 briefly outlines some related work while Section 3 presents the terminology and notations used throughout the paper. Section 4 specifies the CTSPAV and presents a mixed-integer programming (MIP) model that formalizes the CTSPAV. Section 5 describes the column-generation procedure. Section 6 sketches a DARPbased procedure that is used for comparison purposes. Section 7 presents detailed computational results and examines the performance of the proposed approach for servicing the commuting needs of the community under study. Finally, Section 8 provides some concluding remarks.

\section{Related Work}

Both the CTSP and the CTSPAV are generalizations of the Vehicle Routing Problem with Time Windows (VRPTW). The VRPTW seeks a set of minimum cost routes, each departing from and returning to a designated depot, that serve a set of customers, each with a capacity demand and a time window within which service may commence. The problem must ensure that each customer is served exactly once within their time windows while not allowing the vehicle capacities to be exceeded. It is well known to be NP-hard, as Savelsbergh (1985) showed that finding a feasible solution to the problem for a fixed vehicle count is strongly NP-complete. It has been studied extensively in the literature, and numerous methods have been suggested to tackle its complexity, from metaheuristics like Taillard et al. (1997) and Bräysy and Gendreau (2005), to exact solution methods using Lagrangian relaxation (Kohl and Madsen, 1997; Kallehauge et al., 2006), column generation (Desrosiers et al., 1984; Desrochers et al., 1992), or polyhedral approaches (Kohl et al., 1999, Bard et al. 2002; Kallehauge et al. 2007). See Cordeau et al. (2002) for an extensive review of the topic.

The VRPTW was generalized to the Pickup and Delivery Problem with Time Windows (PDPTW) by Dumas et al. (1991), whereby service locations come in pairs, a pickup and a 
delivery location for each customer, that must be serviced in order by the same route. They proposed a dynamic-programming, label-setting algorithm to search for routes that satisfy the new pairing and precedence constraints along with the existing time-window and vehicle-capacity constraints. The algorithm is incorporated within a column-generation procedure to solve the problem. A similar approach was also adopted by Ropke and Cordeau (2009) in their branchand-cut-and-price algorithm for the PDPTW, while Ruland and Rodin (1997) used a polyhedral approach to solve the version of the problem without time-window constraints. The DARP generalizes the PDPTW by introducing ride-duration constraints which limit the maximum duration between each pickup and delivery location pair. It models the maximum time spent on the vehicle by every customer and is critical for guaranteeing a quality-of-service level for services that transport passengers instead of merchandise, like door-to-door transportation services for the disabled and the elderly or those for ridesharing. The problem has also been extensively reviewed by Cordeau and Laporte (2003a) and Cordeau and Laporte (2007), and it has been tackled with methods ranging from approximate methods like heuristics (Bodin and Sexton, 1986; Jaw et al. 1986) and metaheuristics (Cordeau and Laporte, 2003b; Ritzinger et al. 2016), to exact ones utilizing cutting plane methods (Cordeau, 2006) and column generation (Gschwind and Irnich 2015).

Of the many solution approaches proposed for the various generalizations of the VRPTW, column generation is perhaps the most popular due to its elegance in only considering routes that can improve the objective function, and its proven effectiveness in producing strong lower bounds to the problem objective when used in conjunction with the Dantzig-Wolfe decomposition (Dantzig and Wolfe, 1960). It entails a pricing subproblem which searches for routes satisfying problem-specific feasibility constraints, a problem that is typically an Elementary Shortest Path Problem with Resource Constraints (ESPPRC), whereby resource contraints are used to model the feasibility constraints, and the elementarity requirement ensures that each customer is serviced exactly once. Unfortunately, the ESPPRC has been proven to be NP-hard in the strong sense by Dror (1994), and while exact solution methods have been proposed for the problem, e.g., Feillet et al. (2004); Chabrier (2006); Boland et al. (2006); Drexl (2013), the elementarity requirement is more commonly relaxed to produce a Shortest Path Problem with Resource Constraints (SPPRC) which admits a pseudo-polynomial solution approach. A variety of strategies are then adopted to handle non-elementary paths, e.g., Desrosiers et al. (1984), Dumas et al. (1991), Ropke and Cordeau (2009), and Gschwind and Irnich (2015) eliminate them by either preventing their selection in an integer solution or by using infeasible path elimination constraints in the master problem, while Desrochers et al. (1992) and Irnich and Villeneuve (2006) take a middle-ground approach by eliminating 2 - and $k$-cycles from discovered paths respectively. Regardless of whether an SPPRC or an ESPPRC is used in the pricing subproblem, they are typically solved via dynamic programming, the most popular being the generalized label-setting algorithm for multiple resource constraints by Desrochers (1988). Other suggested dynamic-programming approaches include the label-correcting algorithm by Desrosiers et al. (1983) which is based on the Ford-Bellman-Moore algorithm and the label-setting algorithm by Desrochers and Soumis (1988) which generalizes Dijkstra's algorithm. Methods utilizing Lagrangian relaxation (Beasley and Christofides, 1989, Borndörfer et al. 2001), constraint programming (Rousseau et al., 2004), and cutting planes (Drexl, 2013) have also been proposed, and Irnich and Desaulniers (2005) provides an in-depth review of the SPPRC.

More recently, an increased awareness for sustainability of passenger transportation systems combined with the availability of large-scale, real-world trip datasets has shifted the focus towards optimization of car-pooling and ride-sharing services to reduce traffic congestion and pol- 
lution. Baldacci et al. (2004) studied the Car-Pooling Problem (CPP) which seeks to minimize the number of private cars used for commuting to a common workplace. They considered a variant of the problem which optimizes car pooling for the trips to the workplace independently from those for the return trips and assumes that the set of drivers and passengers are known beforehand, making it a specialization of the DARP. The effectiveness of their proposed Lagrangian column-generation method was demonstrated on instances derived from real-world data provided by a research institution in Italy. Agatz et al. (2011) contrasts the CPP with the dynamic ridesharing problem, whereby the latter matches drivers and riders for single, non-recurring trips in real time. They proposed an optimization method which casts the problem as a graph matching problem and solves it at regular intervals within a rolling-horizon framework. They also presented a case study which applies the approach on real-world travel demand data from metro Atlanta. Santi et al. (2014) introduced the notion of shareability graphs as a tool to quantify the potential benefits of ridesharing, and applied it on trip data from the New York City (NYC) Taxi and Limousine Commission trip record which stores information of more than one billion taxi rides in NYC recorded since January 2009. Alonso-Mora et al. (2017) then built on this idea to propose an anytime optimal algorithm for the on-demand ride-sharing problem, and the efficacy of their method was also demonstrated through its application on the trips from the NYC taxi dataset. Agatz et al. (2012) discusses the different planning considerations for and the issues arising from dynamic ridesharing by classifying the different variations of the problem and reviewing the optimization approaches proposed for them. Mourad et al. (2019) takes a broader view of shared mobility in their survey, whereby applications which combine transportation of people and freight in both pre-arranged and real-time settings are reviewed together with their corresponding optimization approaches.

The advent of self-driving technology combined with the race to achieve full driving automation has also triggered a growing interest in Shared Autonomous Vehicle (SAV) systems. Advances in Shared Mobility Services (SMS) and AV technology are widely considered to be mutually beneficial, as the widespread adoption of AVs in SMS could help make AVs financially viable (Gurumurthy and Kockelman, 2018; Stocker and Shaheen, 2019) and accelerate the proliferation of SMS (Thomas and Deepti 2018) at the same time. The potential impact of SAV services, ranging from their effect on the economy and the environment to the changes in policy necessary for their governance, have also been widely discussed and reviewed in works like Milakis et al. (2017), Soteropoulos et al. (2019), and Narayanan et al. (2020). Narayanan et al. (2020) also proposed classifying SAV services as either on-demand or reservation-based systems, according to the time frame within which the trip requests are made. The former allows requests to be made in real time, making it better suited for serving dynamic trips, whereas the latter requires requests to be made in advance, making it better for recurring trips. Each has its own set of advantages: While on-demand systems can address dynamically changing trip demand, reservation-based systems can further reduce the fleet size and increase the efficiency of routes (by reducing empty cruising time and increasing the number of customers served per vehicle), as demonstrated by Wang et al. (2014), as they know the requests ahead of time and can optimize trips over a longer time horizon. Several optimization approaches have been proposed for both systems. For on-demand systems, Farhan and Chen (2018) proposed a three-step approach for optimizing a fleet of SAVs that serves on-demand trips. It first discretizes the time horizon into 5-minute intervals, clusters trip requests from each interval by assigning the riders to their nearest vehicle, and finds the optimal vehicle routes by modeling the requests from each cluster as a VRPTW and solving the problem using a tabu-search metaheuristic. On the other hand, Pinto et al. (2020) considered integrating SAVs with an existing public transit system to better serve 
lower density areas using a bi-level modeling framework to jointly optimize the transit network schedule together with the sizing of the AV fleet. They proposed an iterative heuristic which solves a transit network frequency setting problem using a non-linear solver in the upper level and solves a dynamic combined mode choice-traveler assignment problem using an agent-based simulation in the lower level. For reservation-based systems (which are similar to the system proposed in this study), Ma et al. (2017) proposed an approach to optimize a fleet of SAVs for trips requests that are known ahead of time. However, their approach only allows vehicle sharing whereby each trip is served without being interrupted by other trip requests. This restriction admits an LP model for the problem which can then be solved efficiently. The modeling technique, however, is not applicable to ridesharing problems like to one considered in this study. Bongiovanni et al. (2019) considers a variant of the DARP that uses electric autonomous vehicles, called the e-ADARP. It extends the classical DARP by incorporating additional considerations, like battery management and intermediate stops for vehicle recharging, that only apply to the operation of electric AVs. They proposed two- and three-index formulations for the problem which are solved using a traditional branch-and-cut approach which incorporates new, problem specific valid inequalities. They demonstrated the approach's ability to produce optimal solutions for instances with up to 40 trip requests. Our study, however, considers instances that are five times larger and would therefore require a more robust approach. Numerous other works have touted the potential benefits of these SAV systems, from reducing traffic (Martinez and Viegas, 2017, Alazzawi et al. 2018, Salazar et al., 2018), to increasing road capacity (Friedrich, 2015; Tientrakool et al. 2011; Talebpour and Mahmassani. 2016, Mena-Oreja et al., 2018; Olia et al. 2018), to reducing parking demand (Zhang et al., 2015; Dia and Javanshour, 2017, Zhang and Guhathakurta, 2017). However, there also appears to be a consensus that the benefits require AV adoption reaching a critical mass before they can be truly realized.

Hasan et al. (2018) introduced community-based trip sharing, in which they investigated the efficacy of several optimization models which utilize different sets of driver and passenger matching conditions to optimize car pooling and car sharing for commuting and considered a community-based partitioning approach which clusters commuters based on their residential neighborhoods. They concluded that commuter matching flexibility, i.e., their willingness to adopt different roles and to be matched with different drivers and passengers daily, is critical for an effective trip-sharing platform. This work was extended by Hasan et al. (2020), whereby the best performing car-pooling model from their previous work, which selects the optimal set of drivers from the set of commuters and optimizes their inbound and outbound routes on a daily basis to reduce vehicle count and their total travel distance, is formalized as the CTSP. They then proposed two exact solution approaches for the problem: A method which first searches for all feasible routes and then optimizes their selection using an integer program, and a branchand-price algorithm which searches for feasible routes using column generation. Evaluations on a large-scale, real-world commute-trip dataset from the city of Ann Arbor, Michigan, revealed their capability to reduce vehicle count by up to 57\%. Hasan and Van Hentenryck (2020) then considered special variants of the CTSP in which uncertainties are associated with the schedules of return trips, and proposed a stochastic optimization approach which uses scenario sampling to effectively solve versions of the problem whereby commuters confirm their return times by a fixed deadline or in real time. This paper considers another natural extension to the CTSP. It specifically aims at investigating the potential of autonomous vehicles to address a key shortcoming of the CTSP: the short routes induced by its driver constraints that limit the effective use of the vehicles. Access to the Ann Arbor commute trip dataset and the results of the original CTSP on the trips from the dataset allows this work to be uniquely poised to compare and contrast the 
efficacy of different optimization approaches for conventional and autonomous vehicles, and to provide new insights into the benefits afforded by autonomous vehicles for large-scale sharing of commute trips.

\section{Preliminaries}

This section defines the main concepts used in this paper: trips, mini routes, and AV routes. It also describes the constraints that mini routes and AV routes must satisfy. This work assumes the utilization of a homogeneous fleet of vehicles with capacity $K$ to serve all rides, and that the triangle inequality is satisfied for all travel times and distances.

Trips. A trip $t=\{o, d t, d, a t\}$ is a tuple that consists of an origin $o$, a departure time $d t$, a destination $d$, and an arrival time at of a trip request. Every day, a commuter $c$ makes two trips: a trip $t_{c}^{+}$to the workplace and a return trip $t_{c}^{-}$back home. These trips are called inbound and outbound trips respectively.

Mini Routes. A mini route $r$ is a sequence of locations that visits each origin and destination from a set of inbound or outbound trips exactly once. Let $C_{r}$ denote the set of riders served in $r$. A mini route $r$ must respect the vehicle capacity, i.e., $\left|C_{r}\right| \leq K$, and consists of three phases: a pickup phase where the passengers are picked up, a transit phase where the vehicle travels to the destination, and a drop-off phase where all the passengers are dropped off. During the pickup (resp. drop-off) phase, the vehicle visits only origins (resp. destinations), whereas it travels from an origin to a destination in the transit phase. For instance, a possible mini route for a car with $K=4$ serving trips $t_{1}=\left\{o_{1}, d t_{1}, d_{1}, a t_{1}\right\}, t_{2}=\left\{o_{2}, d t_{2}, d_{2}, a t_{2}\right\}$, and $t_{3}=\left\{o_{3}, d t_{3}, d_{3}, a t_{3}\right\}$ is $r=o_{2} \rightarrow o_{1} \rightarrow o_{3} \rightarrow d_{1} \rightarrow d_{2} \rightarrow d_{3}$, and its pickup, transit, and drop-off phases are given by $o_{2} \rightarrow o_{1} \rightarrow o_{3}, o_{3} \rightarrow d_{1}$, and $d_{1} \rightarrow d_{2} \rightarrow d_{3}$ respectively. An inbound mini route $r^{+}$covers only inbound trips and an outbound mini route $r^{-}$covers only outbound trips.

Definition 3.1 (Valid Mini Route). A valid mini route $r$ serving a set $C_{r}$ of riders visits all of its origins, $\left\{o_{c}: c \in \mathcal{C}_{r}\right\}$, before its destinations, $\left\{d_{c}: c \in C_{r}\right\}$, and respects the vehicle capacity, i.e., it has $\left|C_{r}\right| \leq K$.

Let $T_{i}$ denote the time at which service begins at location $i, s_{i}$ the service duration at $i$, pred $(i)$ the location visited just before $i, \tau_{(i, j)}$ the estimated travel time for the shortest path between locations $i$ and $j$, and $\dot{C}_{r}$ the first commuter served on $r$. Commuters sharing rides are willing to tolerate some inconvenience in terms of deviations to their desired departure and arrival times, as well as in terms of their ride durations compared to their individual, direct trips. Therefore, a time window $\left[a_{i}, b_{i}\right]$ is constructed around the desired times and is associated with each pickup location $i$, where $a_{i}$ and $b_{i}$ denote the earliest and latest times at which service may begin at $i$ respectively. Conversely, only an upper bound $b_{j}$ is associated with each drop-off location $j$ as the arrival time at $j$ is implicitly bounded from below by $a_{j}=a_{i}+s_{i}+\tau_{(i, j)}$, where $i$ is the corresponding pickup location for $j$. On top of that, a duration limit $L_{c}$ is associated with each rider $c$ to denote her maximum ride duration.

Definition 3.2 (Feasible Mini Route). A feasible mini route $r$ is valid, has pickup and drop-off times $T_{i} \in\left[a_{i}, b_{i}\right]$ for each location $i \in r$, and ensures the ride duration of each rider $c \in C_{r}$ does not exceed $L_{c}$. 
Determining if a valid mini route $r$ is feasible amounts to solving a feasibility problem defined by the following constraints on $r$.

$$
\begin{array}{ll}
a_{o_{c}} \leq T_{o_{c}} \leq b_{o_{c}} \quad \forall c \in \mathcal{C}_{r} & \\
T_{d_{c}} \leq b_{d_{c}} \quad \forall c \in C_{r} & \\
T_{\text {pred }\left(o_{c}\right)}+s_{\text {pred }\left(o_{c}\right)}+\tau_{\left(\operatorname{pred}\left(o_{c}\right), o_{c}\right)} \leq T_{o_{c}} & \forall c \in \mathcal{C}_{r} \backslash \dot{\mathcal{C}}_{r} \\
T_{\text {pred }\left(d_{c}\right)}+s_{\text {pred }\left(d_{c}\right)}+\tau_{\left(\operatorname{pred}\left(d_{c}\right), d_{c}\right)}=T_{d_{c}} & \forall c \in \mathcal{C}_{r} \\
T_{d_{c}}-\left(T_{O_{c}}+s_{O_{c}}\right) \leq L_{c} \quad \forall c \in C_{r} &
\end{array}
$$

Constraints (1) and (2) are time-window constraints for pickup and drop-off locations respectively, while constraints (3) and (4) describe compatibility requirements between pickup/dropoff times and travel times between consecutive locations along the route. Finally, constraints (5) specify the ride-duration limit for each rider. Note that constraints (3) allow waiting at pickup locations. Moreover, the service starting times on consecutive locations along $r$ are strictly increasing, which ensures that the route is elementary. Numerous algorithms have been proposed for solving this feasibility problem efficiently, e.g. Tang et al. (2010), Haugland and Ho (2010), Firat and Woeginger (2011), and Gschwind and Irnich (2015). In the following, the Boolean function feasible( $r$ ) is used to indicate whether mini route $r$ admits a feasible solution to constraints (1)-(5).

$A V$ Routes. An AV route $\rho=v_{s} \rightarrow r_{1} \rightarrow \ldots \rightarrow r_{k} \rightarrow v_{t}$ is a sequence of $k$ distinct mini routes that starts at a source node $v_{s}$ and ends at a sink node $v_{t}$, both representing a designated depot.

Definition 3.3 (Feasible AV Route). A feasible AV route $\rho$ is one that consists of a sequence of distinct, feasible mini routes and starts and ends at a designated depot.

In other words, for $\rho$ to be feasible, each of its mini routes must be valid and satisfy constraints (1)-(5). Let $\dot{r}$ denote the first location visited on $r$ and $\ddot{r}$ denote the last. Moreover, each mini route $r_{i}(1 \leq i \leq k)$ must satisfy the following constraints:

$$
\begin{aligned}
& T_{v_{s}}+\tau_{\left(v_{s}, \dot{r}_{1}\right)}=T_{\ddot{r}_{1}} \\
& T_{\ddot{r}_{i}}+s_{\ddot{r}_{i}}+\tau_{\left(\ddot{r}_{i}, \dot{r}_{i+1}\right)} \leq T_{\ddot{r}_{i+1}} \\
& T_{\ddot{r}_{k}}+s_{\ddot{r}_{k}}+\tau_{\left(\ddot{r}_{k}, v_{t}\right)}=T_{v_{t}}
\end{aligned} \quad \forall i=1, \ldots, k-1
$$

Constraints (6)-(8) describe compatibility requirements between the beginning/ending service times of consecutive mini routes along $\rho$ and the travel times between them. The constraints, together with (3) and (4), enforce strictly increasing starting times for service on all consecutive locations along $\rho$, therefore ensuring that $\rho$ is elementary.

\section{The Commute Trip Sharing Problem for Autonomous Vehicles}

This section specifies the CTSPAV that seeks a set of AV routes of minimal cost to serve each inbound and outbound trip of a set of commuters $C$ exactly once. Let $n=|C|$ denote the total number of commuters, $\mathcal{P}^{+}=\{1, \ldots, n\}$ and $\mathcal{D}^{+}=\{n+1, \ldots, 2 n\}$ denote the sets of all pickup and drop-off nodes of inbound trips respectively, and $\mathcal{P}^{-}=\{2 n+1, \ldots, 3 n\}$ and $\mathcal{D}^{-}=\{3 n+1, \ldots, 4 n\}$ denote the sets of all pickup and drop-off nodes of outbound trips respectively. Let $\mathcal{P}=\mathcal{P}^{+} \cup \mathcal{P}^{-}$ 
and $\mathcal{D}=\mathcal{D}^{+} \cup \mathcal{D}^{-}$. The nodes have been defined such that the inbound pickup, inbound dropoff, outbound pickup, and outbound drop-off locations of commuter $i$ are represented by nodes $i$, $n+i, 2 n+i$, and $3 n+i$ respectively, and $n+i$ gives the corresponding drop-off node of pickup node $i \in \mathcal{P}$.

Let $\mathcal{G}=(\mathcal{N}, \mathcal{A})$ denote a directed graph with the node set $\mathcal{N}=\mathcal{P} \cup \mathcal{D} \cup\left\{v_{s}, v_{t}\right\}$ containing all pickup and drop-off nodes together with a source and a sink node representing a designated depot. A ride-duration limit $L_{i}$ is associated with each node $i \in \mathcal{P}$. A time window $\left[a_{i}, b_{i}\right]$ and service duration $s_{i}$ are also associated with each node $i \in \mathcal{P} \cup \mathcal{D}$. There are no time-window constraints for the start and end times of any AV route, as it is assumed that the AVs may start and end their routes at any time of the day. In a first approximation, the edge set $\mathcal{A}=\{(i, j): i, j \in \mathcal{N}, i \neq j\}$ consists of all possible edges. A travel time $\tau_{(i, j)}$, a distance $\sigma_{(i, j)}$, and a cost $c_{(i, j)}$ are associated with each edge $(i, j) \in \mathcal{A}$. The sets of all outgoing and incoming edges of node $i$ are denoted by $\delta^{+}(i)$ and $\delta^{-}(i)$ respectively. By definition of $\mathrm{AV}$ routes, the following precedence constraints apply to the set of nodes:

$$
i<n+i<2 n+i<3 n+i \quad \forall i \in \mathcal{P}^{+}
$$

where $i \prec j$ denotes the precedence relation between nodes $i$ and $j$, i.e., the constraint indicating that $i$ must be visited before $j$ on an AV route.

This paper considers two distinct optimization objectives: (1) a lexicographic objective that first minimizes the number of vehicles and then their total travel distance, and (2) a single objective that only minimizes total travel distance.

\subsection{A MIP Model for the CTSPAV}

This section presents a MIP model for the CTSPAV. The MIP formalizes the CTSPAV and is the foundation of the column-generation procedure presented in the next section. It is defined in terms of the set $\Omega$ of all feasible mini routes and the graph $\mathcal{G}$.

The MIP, also referred to as the master problem (MP $\mathrm{CTSPAV}_{\mathrm{V}}$ ), is shown in Figure 1. It uses two sets of binary variables: variable $X_{r}$ indicates whether mini route $r \in \Omega$ is selected and variable $Y_{e}$ indicates whether edge $e \in \mathcal{A}$ is used in the optimal routing plan. It also uses a continuous variable $T_{i}$ to represent the start of service time at node $i \in \mathcal{P} \cup \mathcal{D}$. The model minimizes the total cost of all selected edges. Constraints 111 enforce coverage of each trip by exactly one mini route, while constraints 12 ensure that edges belonging to selected mini routes are selected. Constraints (13) and (14) conserve flow through each pickup and drop-off node while ensuring each is visited exactly once. Constraints $(15)$ and $(16)$ enforce compatibility of service start times and travel times along selected edges by utilizing large constants for $M_{(i, j)}$ and $\bar{M}_{(i, j)}$. Constraints (17) describe ride-duration limits for each trip, while constraints (18) are time-window constraints for all pickup and drop-off nodes.

The lexicographic objective is accomplished using a blended approach that appropriately weights the sub-objectives: it assigns an identical, large fixed cost to each AV route and a variable cost that is proportional to its total distance. Let $\mathcal{R}$ denote the set of all feasible AV routes. The edge costs are then defined as follows:

$$
c_{e}= \begin{cases}\sigma_{e}+100 \cdot \hat{\varsigma}_{\max } & \forall e \in \delta^{+}\left(v_{s}\right) \\ \sigma_{e} & \text { otherwise }\end{cases}
$$




$$
\min \sum_{e \in \mathcal{A}} c_{e} Y_{e}
$$

subject to

$$
\begin{array}{ll}
\sum_{r \in \mathcal{R}: i \in r} X_{r}=1 \quad \forall i \in \mathcal{P} \\
\sum_{r \in \mathcal{L}: e \in r} X_{r}-Y_{e} \leq 0 \quad \forall e \in \mathcal{A} \backslash\left\{\delta^{+}\left(v_{s}\right) \cup \delta^{-}\left(v_{t}\right)\right\} \\
\sum_{e \in \delta^{+}(i)} Y_{e}=1 \quad \forall i \in \mathcal{P} \cup \mathcal{D} & \\
\sum_{e \in \delta^{-}(i)} Y_{e}=1 \quad \forall i \in \mathcal{P} \cup \mathcal{D} & \\
T_{i}+s_{i}+\tau_{(i, j)} \leq T_{j}+M_{(i, j)}\left(1-Y_{(i, j)}\right) & \forall i, j \in \mathcal{P} \cup \mathcal{D} \\
T_{i}+s_{i}+\tau_{(i, j)} \geq T_{j}-\bar{M}_{(i, j)}\left(1-Y_{(i, j)}\right) & \forall i \in \mathcal{P} \cup \mathcal{D}, \forall j \in \mathcal{D} \\
T_{i+n}-\left(T_{i}+s_{i}\right) \leq L_{i} \quad \forall i \in \mathcal{P} & \\
a_{i} \leq T_{i} \leq b_{i} \quad \forall i \in \mathcal{P} \cup \mathcal{D} & \\
X_{r} \in\{0,1\} \quad \forall r \in \Omega & \\
Y_{e} \in\{0,1\} \quad \forall e \in \mathcal{A} &
\end{array}
$$

Figure 1: The CTSPAV Model.

where $\hat{S}_{\max }$ is a constant equal to the length (total distance) of the longest $\mathrm{AV}$ route, i.e.:

$$
\hat{\boldsymbol{S}}_{\max }=\max _{\rho \in \mathcal{R}} \sum_{(i, j) \in \rho} \sigma_{(i, j)}
$$

The fixed cost, $100 \cdot \hat{\zeta}_{\max }$, that is significantly larger than the total length of any AV route and that is also identical for every edge $e \in \delta^{+}\left(v_{s}\right)$ drives the model to first minimize the total flow emanating from the depot, $v_{s}$. This flow is identical to the total number of AV routes, and thus the number of vehicles used in the solution. The variable cost $\sigma_{e}$ of every edge $e$ then drives the model to minimize the total distance of all selected edges. The edge costs defined in (21) therefore accomplish the desired lexicographic ordering of the objective, which first minimizes the number of vehicles used in the solution and then their total travel distance.

Conversely, when the objective is to just minimize the total distance, the edge costs are simply defined by the edge distance, i.e.,

$$
c_{e}=\sigma_{e} \quad \forall e \in \mathcal{A}
$$

The $\mathrm{MP}_{\text {CTSPAV }}$ model can be seen as a scheduling problem that selects and assembles feasible mini routes to form longer, feasible $\mathrm{AV}$ routes that minimize the total cost. The optimal $\mathrm{AV}$ routes are obtained by constructing paths beginning at $v_{s}$ and ending at $v_{t}$. The start and end times (at the depot) of these routes are then obtained by applying equations (6) and (8) respectively. 


\section{A Column-Generation Procedure for the CTSPAV}

This section presents a column-generation approach to find high-quality solutions to the CTSPAV, referred to as the CTSPAV procedure. The column-generation approach builds on the $\mathrm{MP}_{\text {CTSPAV }}$ but addresses its main computational difficulty: the fact that the $\mathrm{MP}_{\mathrm{CTSPAV}}$ assumes that all mini routes have been pre-computed. The column-generation approach implements an iterative process that considers, at each iteration, a subset $\Omega^{\prime} \subseteq \Omega$ of feasible mini routes and solves a restricted master problem, denoted by $\mathrm{RMP}_{\mathrm{CTSPAV}}$, that is defined as the linear relaxation of $\mathrm{MP}_{\mathrm{CTSPAV}}$ over $\Omega^{\prime}$. Using dual information from $\mathrm{RMP}_{\mathrm{CTSPAV}}$, the column-generation algorithm then searches for feasible mini routes with negative reduced costs by solving a pricing subproblem ( $\mathrm{PSP}_{\mathrm{CTSPAV}}$ ). If such mini routes exist, they are added to the restricted master problem. Each iteration thus defines a new restricted master problem over a larger subset of feasible mini routes. The solving of restricted master problems and pricing subproblems is repeated until the pricing subproblem cannot find any feasible mini routes with a negative reduced cost. Upon completion, the optimal objective value of $\mathrm{RMP}_{\mathrm{CTSPAV}}$ converges to $z^{*}$, the optimal objective of the linear relaxation of $\mathrm{MP}_{\mathrm{CTSPAV}}$. Whenever the solution of $\mathrm{RMP}_{\mathrm{CTSPAV}}$ is integral at conver-

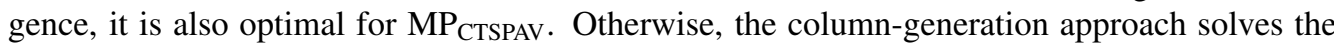
final restricted master problem as a MIP to obtain an integer solution. The objective value of the MIP provides an upper bound to the optimal solution, while the objective value $z^{*}$ of its linear relaxation provides a lower bound. Together, they are used to compute an optimality gap for the integer solution.

The approach adopted in this procedure, which has its pricing subproblem search for feasible mini routes which are then chained together in the master problem to form longer, feasible AV routes that depart from and return to the depot, is unlike the classical column-generation approach adopted in most literature on the VRPTW (e.g. Desrosiers et al.(1984); Desrochers et al.(1992)), PDPTW (e.g. Dumas et al. (1991); Ropke and Cordeau (2009)), or DARP (e.g. Gschwind and Irnich (2015))). The classical approach is an application of the Dantzig-Wolfe decomposition on an edge-flow formulation of the problem: it produces a set-partitioning/covering master problem that just selects feasible routes from a set to ensure every customer is served in the solution. Its pricing problem is then solely responsible for searching for complete feasible routes that originate from and return to the depot (i.e., routes that would correspond to the AV routes of the CTSPAV). In contrast, our approach shifts part of the burden of constructing the AV routes, which are anticipated to be very long, to the master problem instead of completely relegating the task to the pricing subproblem as is commonly done in the classical approach.

\subsection{The Pricing Subproblem}

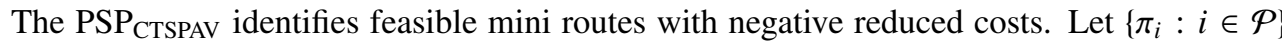
and $\left\{\mu_{e}: e \in \mathcal{A} \backslash\left\{\delta^{+}\left(v_{s}\right) \cup \delta^{-}\left(v_{t}\right)\right\}\right\}$ denote dual values associated with constraints (11) and [12)

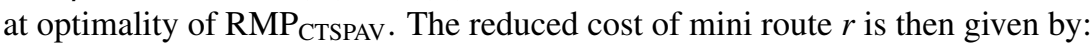

$$
\bar{c}_{r}=-\sum_{i \in r: i \in \mathcal{P}} \pi_{i}-\sum_{e \in r} \mu_{e}
$$

The column-generation approach attempts to generate multiple feasible mini routes during each

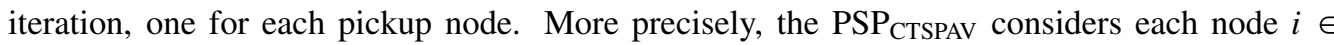
$\mathcal{P}^{+} \cup \mathcal{P}^{-}$as the starting point of a mini route. For each $i \in \mathcal{P}^{+} \cup \mathcal{P}^{-}$, it searches for a mini route $r_{i}$ with minimal reduced cost and selects those with negative reduced costs to augment 
$\Omega^{\prime}$. It accomplishes this by first constructing $2 n$ graphs, $\mathcal{G}_{i}^{+}\left(i \in \mathcal{P}^{+}\right)$and $\mathcal{G}_{i}^{-}\left(i \in \mathcal{P}^{-}\right)$. It then searches for a least-cost path from $i$ to a designated sink node that satisfies all mini-route feasibility constraints from each graph. The complete details of this procedure are given in Appendix A

\subsection{Practical Implementation Considerations}

This subsection reviews a number of important implementation techniques for the CTSPAV column-generation procedure.

Filtering of Graph $\mathcal{G}$. Many edges in $\mathcal{G}$ do not belong to any feasible AV route and can be removed from $\mathcal{A}$. The following sets of infeasible edges are obtained by pre-processing timewindow, pairing, precedence, and ride-duration limit constraints on $\mathcal{A}$ using a combination of rules proposed by Dumas et al. (1991) and Cordeau (2006):

(a) Direct trips to and from the depot:

- $\left\{\left(v_{s}, v_{t}\right),\left(v_{t}, v_{s}\right)\right\}$

- $\left\{\left(i, v_{s}\right),\left(i, v_{t}\right),\left(v_{t}, i\right): i \in \mathcal{P}\right\}$

- $\left\{\left(v_{s}, i\right),\left(i, v_{s}\right),\left(v_{t}, i\right): i \in \mathcal{D}\right\}$

(b) Pairing and precedence of pickup and drop-off nodes of inbound and outbound trips of each commuter (constraints (9)): $\{(i, 2 n+i),(i, 3 n+i),(n+i, i),(n+i, 3 n+i),(2 n+i, i),(2 n+i, n+$ $\left.i),(3 n+i, i),(3 n+i, n+i),(3 n+i, 2 n+i): i \in \mathcal{P}^{+}\right\}$

(c) Time windows along each edge: $\left\{(i, j):(i, j) \in \mathcal{A} \backslash\left\{\delta^{+}\left(v_{s}\right) \cup \delta^{-}\left(v_{t}\right)\right\} \wedge a_{i}+s_{i}+\tau_{(i, j)}>b_{j}\right\}$

(d) Ride-duration limit of each commuter: $\left\{(i, j),(j, n+i): i \in \mathcal{P} \wedge j \in \mathcal{P} \cup \mathcal{D} \wedge i \neq j \wedge \tau_{(i, j)}+\right.$ $\left.s_{j}+\tau_{(j, n+i)}>L_{i}\right\}$

(e) Time windows and ride-duration limits of pairs of trips:

- $\{(i, n+j): i, j \in \mathcal{P} \wedge i \neq j \wedge \neg$ feasible $(j \rightarrow i \rightarrow n+j \rightarrow n+i)\}$

- $\{(n+i, j): i, j \in \mathcal{P} \wedge i \neq j \wedge \neg$ feasible $(i \rightarrow n+i \rightarrow j \rightarrow n+j)\}$

- $\{(i, j): i, j \in \mathcal{P} \wedge i \neq j \wedge \neg$ feasible $(i \rightarrow j \rightarrow n+i \rightarrow n+j) \wedge \neg$ feasible $(i \rightarrow j \rightarrow$ $n+j \rightarrow n+i)\}$

- $\{(n+i, n+j): i, j \in \mathcal{P} \wedge i \neq j \wedge \neg$ feasible $(i \rightarrow j \rightarrow n+i \rightarrow n+j) \wedge \neg$ feasible $(j \rightarrow$ $i \rightarrow n+i \rightarrow n+j)\}$

Note that the sets of edges in (e) utilize the feasible function to determine if a partial route satisfies time-window and ride-duration limit constraints. For instance, the first condition indicates that edge $(i, n+j)$ is infeasible if route $j \rightarrow i \rightarrow n+j \rightarrow n+i$ is infeasible. Figure 2 illustrates an example of graph $\mathcal{G}$ resulting from the removal of the infeasible edges.

Big- $M$ Constants. The RMP $\mathrm{CTSPAV}_{\mathrm{C}}$ utilizes big- $M$ constants in constraints $(15)$ and $(16)$ to enforce the underlying constraints only on selected edges. To ensure that the constants are large enough to accomplish this goal while not being excessively large so as to introduce numerical issues, they are defined as follows:

$$
\begin{array}{ll}
M_{(i, j)}=\max \left\{0, b_{i}+s_{i}+\tau_{(i, j)}-a_{j}\right\} & \forall i, j \in \mathcal{P} \cup \mathcal{D} \\
\bar{M}_{(i, j)}=\max \left\{0, b_{j}-a_{i}-s_{i}-\tau_{(i, j)}\right\} & \forall i \in \mathcal{P} \cup \mathcal{D}, \forall j \in \mathcal{D}
\end{array}
$$

When the lexicographic objective is considered, edge costs defined in 21) uses $\hat{\varsigma}_{\max }$ which is a constant representing the length of the longest AV route. Since enumeration of all feasible $\mathrm{AV}$ routes in $\mathcal{R}$ is impractical, a conservative overestimate is used for $\hat{\boldsymbol{S}}_{\max }$ to accomplish the lexicographic ordering of the sub-objectives. 


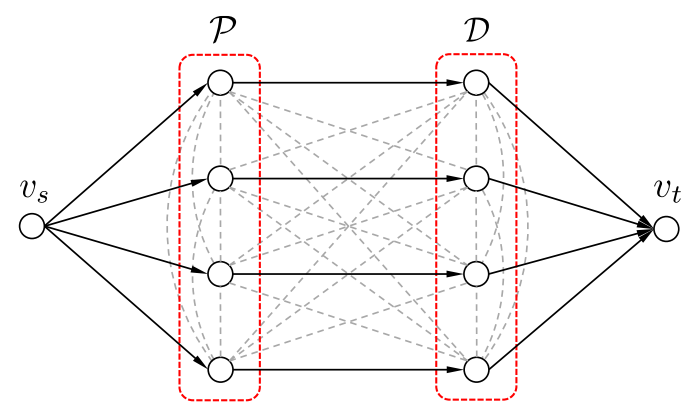

Figure 2: Graph $\mathcal{G}$ (Each Dotted Line Represents a Pair of Bidirectional Edges).

Lower Bound. Column-generation procedures are known to have a tailing-off effect, whereby the rate-of-change of the RMP ${ }_{\text {CTSPAV }}$ objective value $z_{\mathrm{RMP}_{\mathrm{CTSPAV}}}$ progressively decreases as $z_{\mathrm{RMP}} \mathrm{P}_{\mathrm{CTSAV}}$ approaches $z^{*}$ (Lübbecke and Desrosiers, 2005). To mitigate this effect, a dual lower bound to $z^{*}, z_{\mathrm{LB}}$, is defined using the generalized version of the Lasdon bound (Lasdon, 1970), i.e.,

$$
z_{\mathrm{LB}}=\kappa \bar{c}_{r}^{*}
$$

where $\kappa$ is an upper bound to the number of selected mini routes in $\mathrm{MP}_{\mathrm{CTSPAV}}, \kappa \geq \sum_{r \in \Omega} X_{r}$,

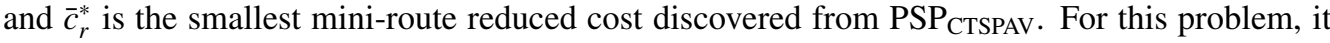
is sufficient to take $\kappa=2 n$. Since the edge costs are all integral, the optimal objective value of $\mathrm{MP}_{\mathrm{CTSPAV}}$ must also be integral, and therefore the column-generation iterations can be terminated when $\left\lceil z_{\mathrm{RMP}_{\text {CTSPAV }}}\right\rceil-z_{\mathrm{LB}}<1$.

Solving the Subproblem. The label-setting algorithm of Gschwind and Irnich (2015) that is used to solved the pricing subproblem produces an intermediate set of non-dominated, feasible mini routes, $\hat{\Omega}_{i}$ for each graph $\mathcal{G}_{i}^{+}\left(i \in \mathcal{P}^{+}\right)$and $\mathcal{G}_{i}^{-}\left(i \in \mathcal{P}^{-}\right)$. Instead of considering only the least-cost route from $\hat{\Omega}_{i}$, all routes from $\hat{\Omega}_{i}$ with negative reduced costs are selected and introduced into $\Omega^{\prime}$ to further accelerate the column-generation convergence. Moreover, since the mini-route search procedure on all graphs are independent, they are solved concurrently in our implementation. Finally, $\Omega^{\prime}$ is initialized with the set of all direct-trip routes, i.e., it is initialized with $\{i \rightarrow n+i$ : $i \in \mathcal{P}\}$

\section{The DARP Column-Generation Procedure}

The CTSPAV can be viewed as a specialization of the DARP: It can be converted into a DARP simply by setting the time-window of each vehicle at the depot to $\pm \infty$. This section describes a column-generation procedure, referred to as the DARP procedure, derived from the algorithm for solving the DARP by Gschwind and Irnich (2015). It is the algorithm to which the CTSPAV procedure is compared in the computational results section. At a high level, the DARP procedure is similar to the CTSPAV procedure as they both use column generation. However, the DARP procedure fundamentally differs from the CTSPAV procedure in that it adopts the classical column-generation procedure. More specifically, the DARP procedure uses a setcovering restricted master problem $\mathrm{RMP}_{\mathrm{DARP}}$ that only selects $\mathrm{AV}$ routes from a set $\mathcal{R}^{\prime}$ to ensure every trip is covered in the solution. Columns of $\mathrm{RMP}_{\mathrm{DARP}}$ represent $\mathrm{AV}$ routes whereas those 


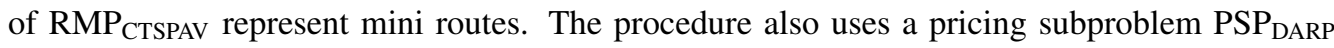
that searches for feasible $\mathrm{AV}$ routes to augment $\mathcal{R}^{\prime}$. Upon convergence of the column-generation process, the RMP ${ }_{\text {DARP }}$ is solved as a MIP to obtain an integer solution 1

\subsection{The Master Problem}

The master problem MP $\mathrm{DARP}_{\text {is }}$ a set-covering formulation that seeks the optimal routing plan for the CTSPAV. It is defined on the set of all feasible AV routes $\mathcal{R}$ and uses a binary variable $X_{\rho}$ that indicates whether route $\rho \in \mathcal{R}$ is used in the plan. The model is listed in 28)-(30).

$$
\begin{aligned}
& \min \sum_{\rho \in \mathcal{R}} c_{\rho} X_{\rho} \\
& \text { subject to } \\
& \sum_{\rho \in \mathcal{R}} a_{i, \rho} X_{\rho} \geq 1 \quad \forall i \in \mathcal{P} \\
& X_{\rho} \in\{0,1\} \quad \forall \rho \in \mathcal{R}
\end{aligned}
$$

The objective function (28) minimizes the total cost of the selected routes. Constant $a_{i, \rho}$ in constraints 29 represents the number of times node $i$ is visited by route $\rho$. These constraints ensure that each pickup node is covered in the optimal plan. Our experimental evaluations indicated that a set-covering formulation produces stronger integer solutions than a set-partitioning formulation.

To find a routing plan that minimizes vehicle count, the cost $c_{\rho}$ of each route is set to 1 . On the other hand, to find a plan minimizing total travel distance, the cost $c_{\rho}$ is set to the total distance of $\rho$, i.e., $c_{\rho}=\sum_{(i, j) \in \rho} \sigma_{(i, j)}$. Finally, to implement a lexicographic objective that first minimizes vehicle count and then their total distance, the model is solved twice. The model is first solved to produce the optimal vehicle count $\chi_{\mathrm{MIP}}^{*}$. The constraint

$$
\sum_{\rho \in \mathcal{R}} X_{\rho}=\chi_{\mathrm{MIP}}^{*}
$$

is then introduced to the model to fix its vehicle count to its optimal value. The model is then solved again to optimize the secondary objective.

While a blended approach similar to that used in the CTSPAV procedure could have also been used here to implement the lexicographic objective, initial experimental evaluations revealed that the greater complexity of $\mathrm{PSP}_{\mathrm{DARP}}$, which is significantly more expensive than $\mathrm{PSP}_{\mathrm{CTSPAV}}$, combined with the use of the Lasdon bound (27) results in a column-generation phase that converges significantly slower. The proposed multi-objective approach, which first just minimizes the vehicle count and therefore uses identical costs for the routes of RMP ${ }_{\mathrm{DARP}}$ (unlike route costs for the blended approach), permits the use of the dual bound proposed by Farley $(1990)$ in the columngeneration phase which is stronger than the Lasdon bound in this setting. This stronger dual bound consequently allows the column-generation termination criterion to be satisfied earlier, thus resulting in a faster converging column-generation phase for the primary objective. And

\footnotetext{
${ }^{1}$ The branch-and-price approach proposed by Gschwind and Irnich 2015 , is not considered because it is found to be too expensive for the problem instances used in this work. Even the root node of the branch-and-price tree cannot be solved within the allocated time budget for the real instances considered in this paper.
} 
while a similar approach could have also been used for the CTSPAV procedure, the less expen-

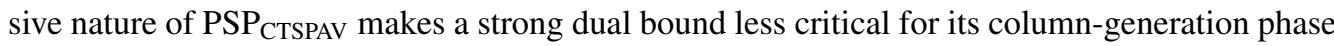
which already converges quickly. In the end, the blended and the multi-objective approaches are different yet valid alternatives for implementing the lexicographic objective. The latter, which applies the lexicographic ordering directly, is preferred for the DARP procedure simply because it allows the column-generation phase for the primary objective to converge more quickly in practice and is seen as a necessity to counteract the increased complexity of its pricing subproblem.

\subsection{The Pricing Subproblem}

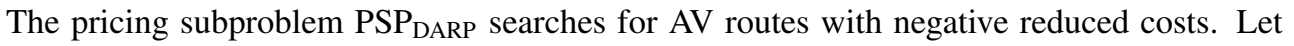
$\left\{\alpha_{i}: i \in \mathcal{P}\right\}$ denote the set of optimal duals of constraints (29) and $\beta$ be that of constraint 31. When RMP $\mathrm{DARP}_{\mathrm{DAs}}$ he vehicle count-minimization objective, the reduced cost of route $\rho$ is given by:

$$
\bar{c}_{\rho}=1-\sum_{i \in \mathcal{P}} a_{i, \rho} \alpha_{i}
$$

When the distance-minimization objective is applied, the reduced cost of $\rho$ is given by:

$$
\bar{c}_{\rho}=\sum_{(i, j) \in \rho} \sigma_{(i, j)}-\sum_{i \in \mathcal{P}} a_{i, \rho} \alpha_{i}
$$

Finally, when constraint (31) is also present in RMP ${ }_{\text {DARP }}$ with the distance-minimization objective, the reduced cost of $\rho$ is given by:

$$
\bar{c}_{\rho}=\sum_{(i, j) \in \rho} \sigma_{(i, j)}-\sum_{i \in \mathcal{P}} a_{i, \rho} \alpha_{i}-\beta
$$

The pricing subproblem searches for routes with negative reduced costs using a graph $\mathcal{G}$ identical to the one described in Section 4 A reduced cost $\bar{c}_{(i, j)}$ is assigned to each edge $(i, j) \in \mathcal{A}$, and it is defined according to the objective function used. For the vehicle count-minimization objective, $\bar{c}_{(i, j)}$ is given by

$$
\bar{c}_{(i, j)}= \begin{cases}1 & \forall(i, j) \in \delta^{+}\left(v_{s}\right) \\ -\alpha_{i} & \forall i \in \mathcal{P}, \forall j \in \mathcal{N} \\ 0 & \forall i \in \mathcal{D}, \forall j \in \mathcal{N}\end{cases}
$$

When the distance-minimization objective is used, $\bar{c}_{(i, j)}$ is given by

$$
\bar{c}_{(i, j)}= \begin{cases}\sigma_{(i, j)}-\alpha_{i} & \forall i \in \mathcal{P}, \forall j \in \mathcal{N} \\ \sigma_{(i, j)} & \forall i \in \mathcal{D} \cup\left\{v_{s}\right\}, \forall j \in \mathcal{N}\end{cases}
$$

The edge reduced costs are defined so that the total cost of any path in $\mathcal{G}$ from $v_{s}$ to $v_{t}$ is equivalent to the reduced cost of the path defined in (32)-(34). In the presence of constraint (31) for the distance-minimization objective, (36) is still used to define edge reduced costs and $-\beta$ is just added to the final path cost to obtain the reduced cost defined in (34).

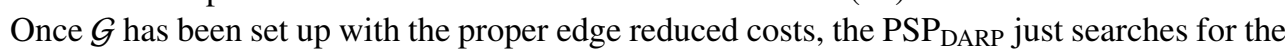
least-cost path from $v_{s}$ to $v_{t}$ that satisfies the time-window, vehicle-capacity, pairing, precedence, 
and ride-duration limit constraints. This least-cost path is then added to $\mathcal{R}^{\prime}$ if the cost is negative. The problem is an SPPRC and is solved using the label-setting dynamic program proposed by Gschwind and Irnich (2015) which utilizes resource constraints to enforce the route-feasibility constraints.

In the presence of negative cost cycles in $\mathcal{G}$, the label-setting algorithm may produce nonelementary paths. Whereas an additional resource may be introduced to the pricing algorithm to guarantee path elementarity, the SPPRC then becomes an ESPPRC which is extremely hard to solve. Therefore, the implementation adopts a strategy from Ropke and Cordeau (2006) and Gschwind and Irnich (2015) which simply relaxes this elementarity requirement. While doing so theoretically causes the RMP ${ }_{\text {DARP }}$ to converge to a weaker primal lower bound as it now admits a larger set of routes $\mathcal{R}^{\prime \prime} \supseteq \mathcal{R}^{\prime}$, both Ropke and Cordeau (2009) and Gschwind and Irnich (2015) have found that the resulting lower bound is only slightly weaker in practice.

\subsection{Implementation Strategies}

Similar to the CTSPAV procedure, a dual lower bound is maintained during the columngeneration procedure to mitigate its tailing-off effect. When the vehicle count-minimization objective is active, the lower bound proposed by Farley (1990) is adopted since the cost of each route is identical. It is given by:

$$
z_{\mathrm{LB}}^{\prime}=\frac{z_{\mathrm{RMP}}{ }_{\mathrm{DARP}}}{1-\bar{c}_{\rho}^{*}}
$$

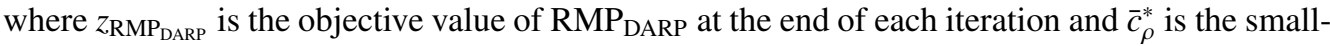
est route reduced cost discovered by $\mathrm{PSP}_{\mathrm{DARP}}$. The column generation is then terminated when $\left\lceil z_{\mathrm{RMP}_{\mathrm{DARP}}}\right\rceil-z_{\mathrm{LB}}^{\prime}<1$. To accomplish the lexicographic objective during the column generation, constraint 31 is introduced to the RMP ${ }_{\mathrm{DARP}}$ after the primary objective has converged with its right-hand side set to $\left\lceil z_{\mathrm{RMP}_{\mathrm{DARP}}}\right\rceil$. The objective function is then switched to distance minimization after which the column generation is resumed. For the distance-minimization objective, the generalized Lasdon bound defined in 27) is used as a dual lower bound, and the column generation is terminated when $\left\lceil z_{\mathrm{RMP}}{ }_{\mathrm{DARP}}\right\rceil-z_{\mathrm{LB}}<1$.

Our implementation also incorporates the interior-point, dual-stabilization method proposed by Rousseau et al. (2007) to accelerate the column-generation convergence. In addition, all non-dominated routes with negative reduced costs resulting from the label-setting algorithm in PSP $_{\text {DARP }}$ are added to the master problem. The column-generation phase is seeded with routes $\left\{i \rightarrow n+i \rightarrow 2 n+i \rightarrow 3 n+i: i \in \mathcal{P}^{+}\right\}$. Since this set of routes represents a feasible solution to the problem, it guarantees the existence of a feasible integer solution to the MIP for both the distance-minimization and the primary lexicographic objective. Recall that for the secondary lexicographic objective, the right-hand side of the introduced constraint 31 is set to $\chi_{\text {MIP }}^{*}$ which represents the objective value of the optimal integer solution for the primary objective. The existence of this feasible integer solution guarantees that one also exists for the MIP with the secondary objective.

Non-elementary routes produced in the column-generation phase are removed prior to solving the $\mathrm{RMP}_{\mathrm{DARP}}$ as a MIP. Repeated nodes are identified from each non-elementary route and only the first instance of each repeated node is preserved in the route (subsequent instances are removed). The resulting route is feasible as the non-elementary version already satisfies the time-window, vehicle-capacity, pairing, precedence, and ride-duration limit constraints. Similarly, since a set-covering formulation is used, a node may be visited by multiple routes in the 


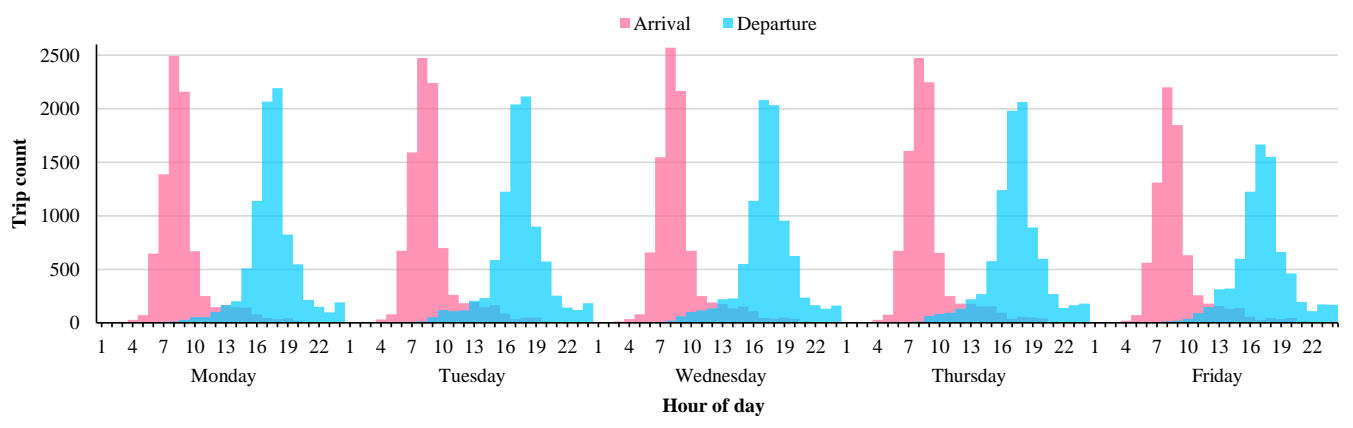

Figure 3: Distribution of Arrival and Departure Times of Commute Trips from Parking Structures over Week 2 of April 2017.

integer solution. This is fixed by simply preserving the node in an arbitrarily selected route and removing it from the others. Since the travel distances satisfy the triangle inequality, this step only shortens the affected routes and hence improves the total travel distance of the solution while maintaining its vehicle count.

\section{Case Study and Experimental Results}

This section evaluates the potential benefits of autonomous vehicles on a real case study. It also reports a variety of experimental results on the efficiency of the optimization algorithms.

\subsection{The Dataset and Construction of Problem Instances}

The performance of the CTSPAV and DARP procedures are evaluated on problem instances derived from the real-world, commute-trip dataset used by Hasan et al. (2018). The dataset consists of daily arrival and departure information of a total of 15,000 commuters traveling to 15 parking structures located in downtown Ann Arbor, Michigan. Collected throughout the month of April 2017, this information includes the exact arrival and departure times of every commuter to and from each parking structure. Joining this information with the addresses of the commuter homes - which are situated within the city as well as its surrounding region, an area that spans 13,000 square miles - yields detailed information about an average of 9,000 commute trips per weekday. The results in this section focus on the busiest days (Monday-Thursday) of the busiest week of the month (week 2). Figure 3 shows the distribution of arrival and departure times of these trips to and from the parking structures over this week. The travel patterns display remarkably consistent distributions over the different weekdays. The arrival and departure times peak at 6-9 am and 4-7 pm respectively, highlighting the typical peak commute hours.

For additional perspectives, the trips are partitioned into two sets: the approximately 2,200 daily trips made by commuters living within Ann Arbor city limits (the region bounded by highways US-23, M-14, and I-94), and the remainder made by commuters living outside the region. The rationale behind this split is to distinguish between the results of trips for commuters living nearer to the downtown area, where the parking structures are located, and those for commuters living further away. The trips from each set are then further partitioned into smaller problem instances using the clustering algorithm from Hasan et al. (2020) which groups at most $N$ commuters together based on the spatial proximity of their home locations. The clusters are thought 
of as "neighborhoods" within which trip sharing is done exclusively. This notion of breaking down a problem into smaller instances is in line with the conclusion by Agatz et al. (2012), that effective decomposition techniques are necessary for the computational feasibility of large-scale problems.

Several assumptions were made to characterize the nature of the requests submitted to the trip-sharing platform. First, each rider $i$, when requesting a trip, specifies a desired arrival time $a t_{i}^{+}$to the inbound trip destination and a desired departure time $d t_{i}^{-}$from the outbound trip origin. This assumption is consistent with those in the literature on the DARP, e.g., Jaw et al. (1986), Cordeau and Laporte (2003b), and Cordeau (2006). Secondly, each rider tolerates a maximum shift of $\pm \Delta$ to the desired times. Therefore, if the arrival and departure times at the parking structure from the dataset are considered as the desired times, then a delivery time upper bound of $b_{i}=a t_{i}^{+}+\Delta$ can be associated with each $i \in \mathcal{D}^{+}$and a pickup time window of $\left[a_{i}, b_{i}\right]=$ $\left[d t_{i}^{-}-\Delta, d t_{i}^{-}+\Delta\right]$ can be associated with each $i \in \mathcal{P}^{-}$. Consequently, a departure time window of $\left[a_{i}, b_{i}\right]=\left[b_{n+i}-s_{i}-L_{i}-2 \Delta, b_{n+i}-s_{i}-L_{i}\right]$ is associated with each $i \in \mathcal{P}^{+}$and a delivery time upper bound of $b_{i}=b_{i-n}+s_{i-n}+L_{i-n}$ is associated with each $i \in \mathcal{D}^{-}$. Finally, each rider $i$ will tolerate at most an $R \%$ extension to her direct-trip ride duration, i.e., $L_{i}=(1+R) \cdot \tau_{(i, n+i)}$ for each $i \in \mathcal{P}$. Hunsaker and Savelsbergh (2002) also used a similar assumption. This use of a single multiplication factor for modeling the maximum tolerable ride-duration (versus a more fine-grained approach) is seen as a practical necessity to cater to the massive volume of trips considered in the case study, and it is seen as a more realistic alternative to the approach used in other works on the DARP (e.g., Cordeau (2006) used an identical duration for every trip). Results of sensitivity analyses on both $\Delta$ and $R$ are provided in later sections to demonstrate their effect on the final results.

Depot Configurations. This paper explores two hypothetical depot configurations: (1) a central depot configuration in which all neighborhoods are served by vehicles from a single, centralized depot, and (2) a local depot configuration whereby each neighborhood is served by a local depot situated within the neighborhood itself. For the first scenario, the largest parking structure from the dataset considered is arbitrarily designated as the central depot. In the second scenario, the home address $l_{c}$ that is nearest to every other location within cluster $\mathcal{P}_{c}$ is selected as the hypothetical local depot location, i.e.,

$$
l_{c}=\underset{i \in \mathcal{P}_{c}}{\arg \min }\left\{\sum_{j \in \mathcal{P}_{c} \backslash\{i\}} \sigma_{(i, j)}+\sigma_{(j, i)}\right\} .
$$

\subsection{Experimental Setup and Parameters}

The GPS coordinates of every address considered are geocoded using Geocodio, while the shortest path, travel time, and travel distance between any two locations are estimated using GraphHopper's Directions API that uses OpenStreetMap data. All algorithms are implemented in $\mathrm{C}++$ with parallelization being handled with OpenMP. The label-setting algorithm of Gschwind and Irnich (2015) is implemented using the resource-constrained shortest path framework from the Boost Graph Library (version 1.70.0), while all LPs and MIPs are solved with Gurobi 8.1.1. Every problem instance is solved on a compute cluster, utilizing 12 cores of a $3.0 \mathrm{GHz}$ Intel Xeon Gold 6154 processor and 32 GB of RAM. A total time budget of 2 hours is allocated for each instance; 1 hour for the column-generation phase and another 1 hour for solving the MIP for the CTSPAV procedure. The same total time budget is allocated for the DARP procedure. However, since initial evaluations showed that it requires more time for the column-generation phase and 


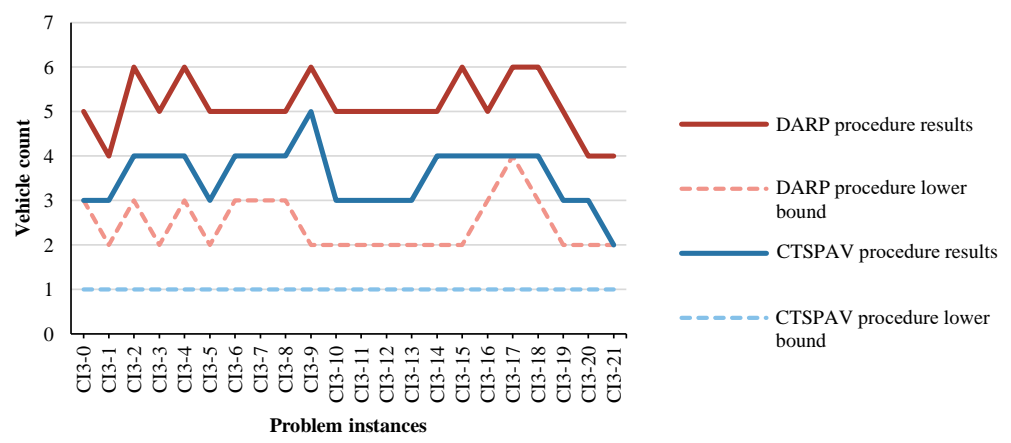

Figure 4: Comparison of Vehicle Count Results for Problem Instances Inside City Limits with a Lexicographic Objective and a Central Depot Configuration.

less for solving the MIP, 1.5 hours is allocated for its column-generation phase and 0.5 hours for solving its MIP. All reported results are from the best feasible solution obtained within the time limit.

The experiments consider the use of autonomous cars and hence $K=4$. Initial empirical evaluations found that $N=100$ for the clustering algorithm produces neighborhoods that are sufficiently large to provide ample opportunities for trip sharing while not producing intractable problem instances. This setting was thus used to generate the problem instances for all experiments. Finally, unless otherwise stated, values of $\Delta=10$ mins and $R=50 \%$ are used in all experiments. Appendix B summarizes results on the optimality gaps and computation times of the procedures. Both the appendix and the next subsection summarizes the results of all problem instances obtained from applying the clustering algorithm on all commute trips from the Wednesday of week 2 (which generated 22 and 68 clusters inside and outside the city respectively).

\subsection{Performance Comparison of the CTSPAV and DARP Procedures}

This section presents a comparison of the results of the CTSPAV procedure against those of the DARP, particularly the final objective values from their MIPs together with the corresponding lower bounds of each procedure for every problem instance considered. Figure 4 compares their vehicle count results when the lexicographic objective and central depot configuration are used on clusters inside the city, whereas Figure 5 does the same for clusters outside. The horizontal axis lists the problem instances while the vertical displays the vehicle counts. Figure 4 shows the typical trend: the DARP procedure produces stronger lower bounds and the CTSPAV procedure produces smaller vehicle counts. This trend carries over to Figure 5, Even though there are a few instances where the DARP procedure outperform the CTSPAV procedure, overall the CTSPAV matches or outperforms the DARP on more than $80 \%$ of the instances shown in the figure. Vehicle count results of instances with the local depot configuration exhibit similar trends and are not shown here.

Table 1 then compares the aggregated vehicle count results from all clusters for the same lexicographic objective and central depot configuration. This comparison is especially important as the results of all clusters are summarized through aggregation in the case study. The table shows a significant difference between the results of the two procedures, with the DARP procedure producing aggregated vehicle counts that are $45 \%$ larger inside the city, $6 \%$ larger outside 


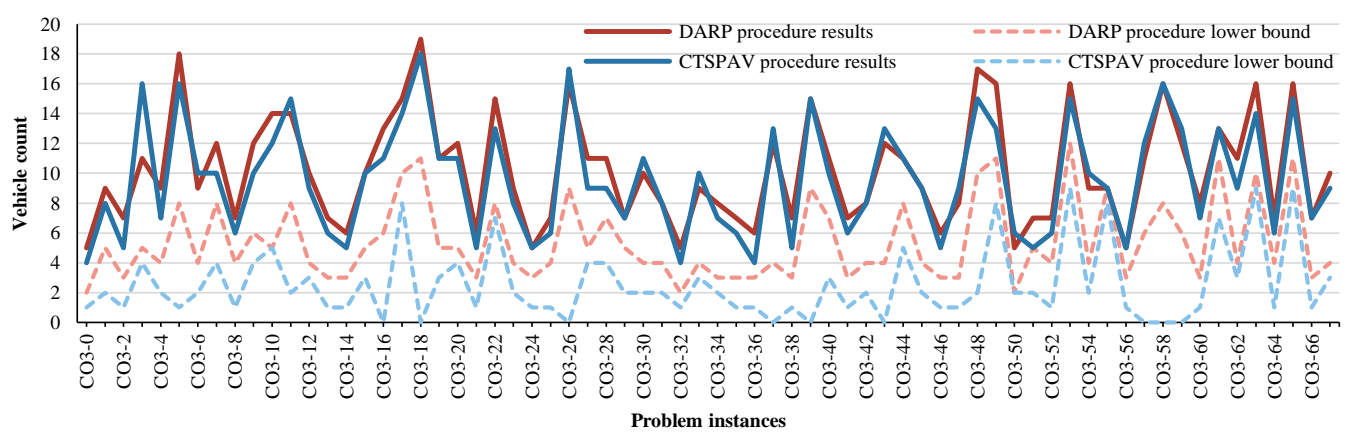

Figure 5: Comparison of Vehicle Count Results for Problem Instances Outside City Limits with a Lexicographic Objective and a Central Depot Configuration.

Table 1: Difference in Aggregate Vehicle Counts of CTSPAV and DARP Procedures

\begin{tabular}{cccc}
\hline \multirow{2}{*}{ Location } & \multicolumn{3}{c}{ Aggregate vehicle count } \\
\cline { 2 - 4 } & CTSPAV procedure & DARP procedure & Percentage difference \\
\hline Inside & 78 & 113 & $+45 \%$ \\
Outside & 652 & 694 & $+6 \%$ \\
Combined & 730 & 807 & $+11 \%$ \\
\hline
\end{tabular}

the city, and $11 \%$ larger overall. This significant difference makes another strong case for using results of the CTSPAV procedure in the case study.

Figures 6 and 7 compare the results when minimizing total distance under a central depot configuration for clusters inside and outside the city respectively. The horizontal axis lists the problem instances while the vertical displays the total distances. For a few clusters outside the city, even a single column-generation iteration of the DARP procedure cannot be completed within the time limit. The comparison for these instances are therefore excluded from Figure 7 . Similar to Figures 4 and 5, the CTSPAV procedure produces stronger total distance results. In fact, it outperforms the DARP procedure in all problem instances considered. In addition, the procedure also appears to produce stronger lower bounds for this objective function. There is a caveat to this observation however. The weak lower bounds of the DARP procedure in this case can be attributed mainly to its column-generation phase not converging within its (longer) time limit. When column-generation does converge, e.g., in instances CO3-51 and CO3-55 in Figure 7, the lower bounds produced are comparable to those of the CTSPAV procedure. The figures also highlight the excellent optimality gap of the CTSPAV procedure for this objective function. Once again, total distance results of instances with the local depot configuration are not summarized here as they display similar trends. Since the CTSPAV procedure consistently produces the stronger final results, it is used to obtain the results for the subsequent case study.

\subsection{Vehicle and Travel Distance Reduction Results}

Figures 8 and 9 show aggregated vehicle count (VC) results of all clusters inside and outside city limits respectively for the first four weekdays of week 2. Each figure shows VC results for every combination of objective function (lexicographic or distance minimization) and depot configuration (central or local) for the CTSPAV: they are labeled "Lex Central", "Dist Central", 


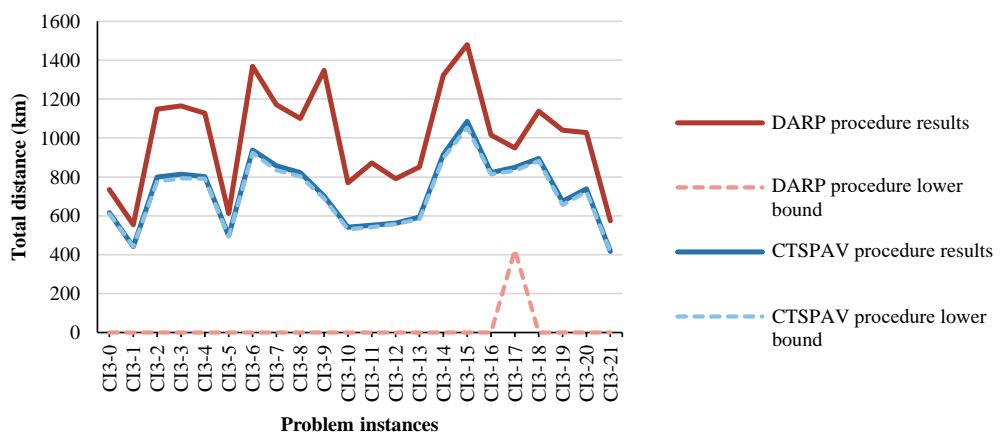

Figure 6: Comparison of Procedure Total Distance Results for Problem Instances Inside City Limits with DistanceMinimization Objective and Central Depot Configuration.

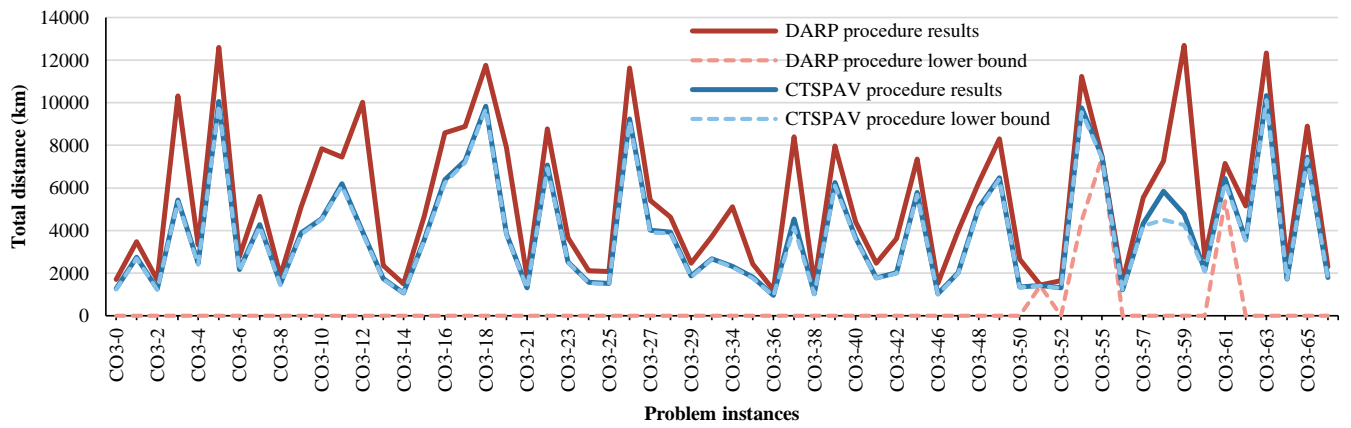

Figure 7: Comparison of Procedure Total Distance Results for Problem Instances Outside City Limits with DistanceMinimization Objective and Central Depot Configuration.

"Lex Local", and "Dist Local" respectively. Each figure also shows VC results of trips under nosharing conditions and of the CTSP described by Hasan et al. (2020) for additional perspective. The percentages of the VCs for each method as a fraction of the no-sharing VC are also included. The figures indicate that the CTSPAV consistently requires fewer vehicles than the CTSP to cover all trips regardless of the location of the clusters, objective function, and depot configuration. This is not surprising, as the CTSPAV addresses the key limitation of the CTSP. As mentioned by Hasan et al. (2020), routes of the CTSP are relatively short as the number of locations they can visit are limited by the ride-duration constraint of their drivers, as well as the time windows at the origins and destinations of the driver trips. In contrast, AVs are not subject to these limitations. Therefore, they can travel back and forth between the parking structures and the neighborhoods to serve trips throughout the day, consequently allowing fewer vehicles to be utilized to cover the same amount of trips. What is striking however is the magnitude of the reduction in the number of vehicles: the VCs are reduced by $96 \%$ and $90 \%$ inside and outside city limits respectively when using $A V s$.

Results for the different objective functions show that the vehicle reduction of the lexicographic objective is significantly better than that of the distance minimization. Again, this result is not surprising as reducing VC is the primary goal of the former objective function, whereas it is not a consideration in the latter. The discrepancy between the vehicle reductions inside and outside city limits can be attributed to the larger distance between the parking structures and the 

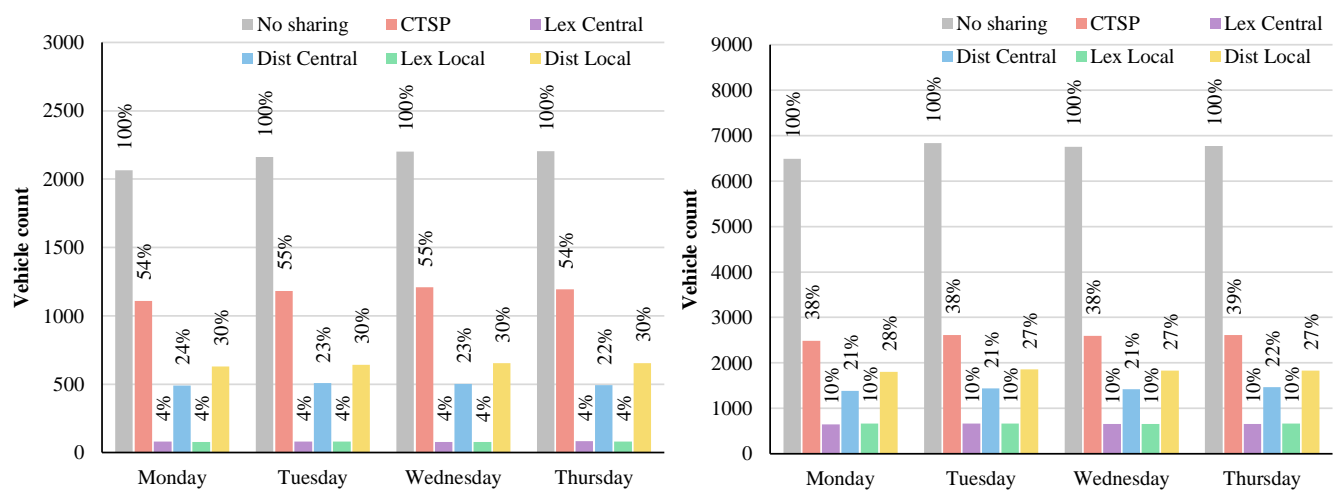

Figure 8: Aggregate Vehicle Count Results from All Clus- Figure 9: Aggregate Vehicle Count Results from All Clusters Inside City Limits. ters Outside City Limits.

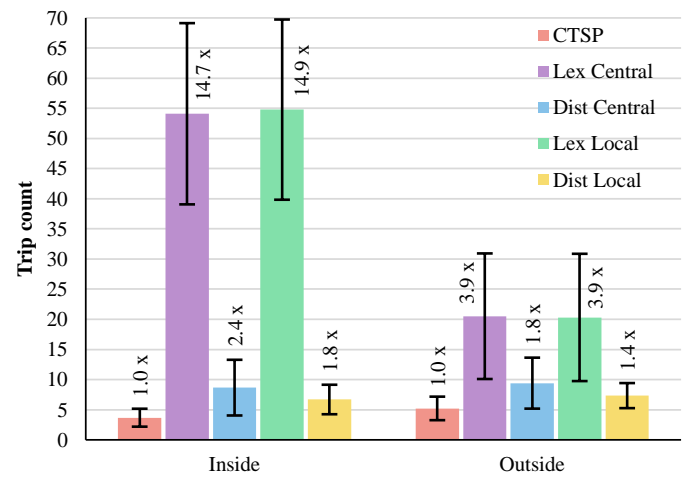

Figure 10: Average Number of Trips Served by Routes of Each Method.

neighborhoods outside city limits. As a result, the AVs serving these neighborhoods spend a larger fraction of their time in the transit phase, therefore limiting the number of trips they could serve in a day. This factor is further highlighted in Figure 10 which summarizes the average number of trips served by the routes of each method. The figure also shows each count as a multiplicative factor of the count for the CTSP, and the error bars depict the standard deviations of the trip counts. For the CTSPAV with the lexicographic objective, the routes from the clusters outside the city visit significantly fewer nodes than those inside the city as their transit phases are longer. However, regardless of the position of the clusters, routes of the CTSPAV consistently cover more trips on average than those of the CTSP. In fact, inside city limits, routes of the CTSPAV with the lexicographic objective serve, on average, an order of magnitude more trips than those of the CTSP.

Figures 11 and 12 summarize the corresponding aggregated vehicle miles traveled (VMT) for all clusters inside and outside city limits respectively. Similar to Figures 8 and 9 they show CTSPAV results for every combination of objective function and depot configuration, as well as results of the CTSP and of trips under no-sharing conditions for additional perspectives. Similarly, the percentages represent the VMT of each method as a fraction of the no-sharing VMT. The VMT percentage of each method outside the city limits is consistently smaller than those 

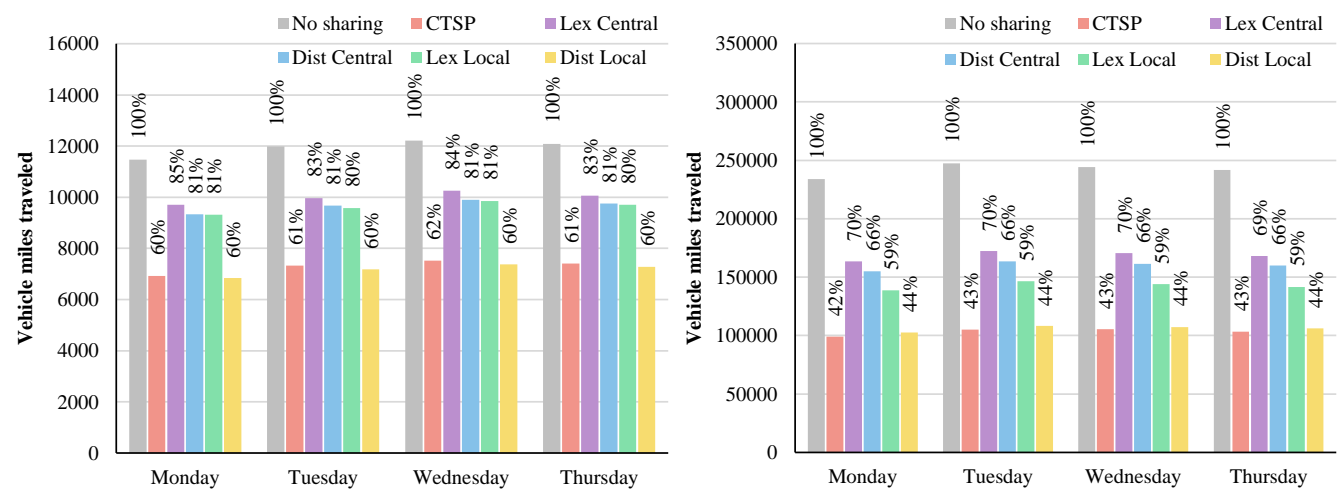

Figure 11: Aggregate Vehicle Miles Traveled from All Figure 12: Aggregate Vehicle Miles Traveled from All Clusters Inside City Limits. Clusters Outside City Limits.

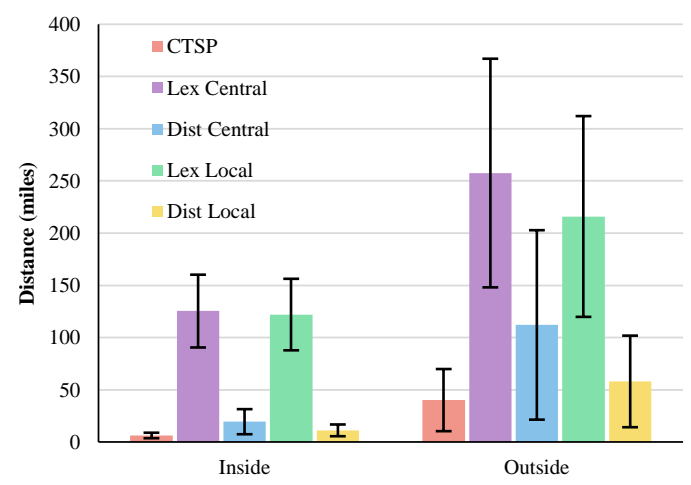

Figure 13: Average Distance Traveled Per Vehicle of Each Method.

inside. This can be attributed to the neighborhoods outside the city being further away from the parking structures.

When a central depot is used for the CTSPAV, VMT is reduced by 15-19\% inside the city (resp., 30-34\% outside the city). Although sizeable, the reduction is not as significant as that of the CTSP, as the AVs have to travel back and forth between the neighborhoods and the parking structures which increases their total travel distance. When the different objective functions for the central depot configuration are compared, the distance-minimization objective only improves VMT by $2-4 \%$ at the expense of significantly larger vehicle counts. VMT is further reduced when local depots are used, as each AV has to travel a shorter distance from the depot to reach its first pickup location and from its last drop-off location back to the depot since both these locations are in the neighborhoods. When the different objective functions for this depot configuration are compared, it can be seen that the distance-minimization objective significantly improves VMT by $15-21 \%$, once again at the expense of a significant increase in vehicle count. In fact, the VMT of the distance-minimization objective is comparable to that of the CTSP. This can be explained by referring to Figure 10, which shows their trip counts to be similar. In other words, the VMT of the CTSPAV with the distance-minimization objective and local depot configuration is comparable to the CTSP because its routes are serving fewer trips per day. 


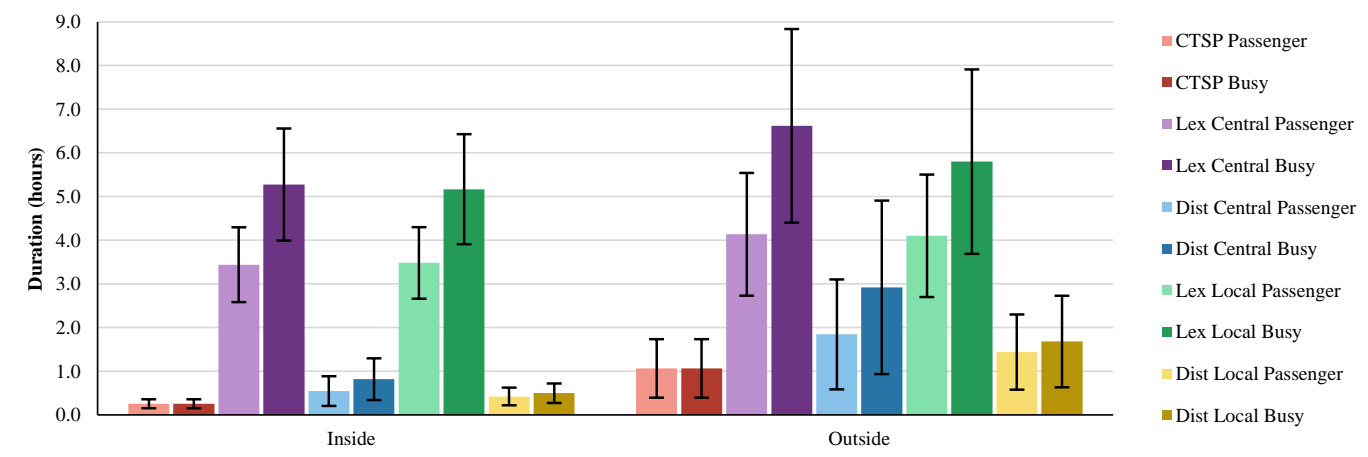

Figure 14: Average Passenger and Busy Ride Durations of the Routes of Each Method.

Figure 13 provides further insight into the daily average of the distance traveled per vehicle for each method. The error bars depict the standard deviations for each method. As expected, average travel distances are larger for clusters outside the city for each method. They are also larger for the CTSPAV when the lexicographic objective is used as the AVs travel back and forth more between the neighborhoods and parking structures in order to reduce vehicle count.

Figure 14 takes a look at the daily average passenger and busy ride durations. The error bars again represent the standard deviations for each method. The passenger duration is defined as the total duration of the day during which at least one passenger is on the vehicle, whereas the busy duration is the passenger duration of a vehicle combined with the duration spent traveling between locations with no passengers. The complement of the busy duration, the idle duration, is therefore the duration of the day during which a vehicle is parked at a depot/at home/at a parking structure, combined with the duration spent waiting to pick up passengers while being empty. The passenger and busy durations of the CTSP are therefore identical as its vehicles are driven by the commuters themselves. In other words, they never travel without any passengers. For the CTSPAV, its busy duration is longer than its passenger duration as the latter is a subset of the former. In fact, the difference between the two represent the duration spent by the vehicle traveling without any passengers. For the lexicographic objective, this duration takes up a large fraction of its busy time, which supports earlier claims that vehicles under this configuration do more back and forth traveling to reduce its vehicle count.

The average durations of each method for clusters outside city limits are longer than corresponding durations inside as the vehicles must travel farther between the neighborhoods and the parking structures. The CTSP also has the shortest passenger and busy durations, which are consistent with the results from Figure 10 which showed the CTSP having the lowest average trip count. For the various configurations of the CTSPAV, the ones utilizing the lexicographic objective produce the largest passenger and busy durations, also consistent with their average trip count results from Figure 10. The results also reveal another drawback of the distanceminimization objective. Not only does the configuration require relatively larger vehicle counts to cover the same number of trips, but its vehicles are less busy throughout the day than those of the lexicographic objective. In other words, the vehicles spend a longer time every day being idle, which somewhat defeats the purpose of utilizing AVs in the first place.

Figures 15 and 16 provide a deeper look into how the passenger durations are spent by the routes of each method for clusters inside and outside city limits respectively. It shows the fraction of the total passenger duration spent serving one, two, three, or four passengers for each 

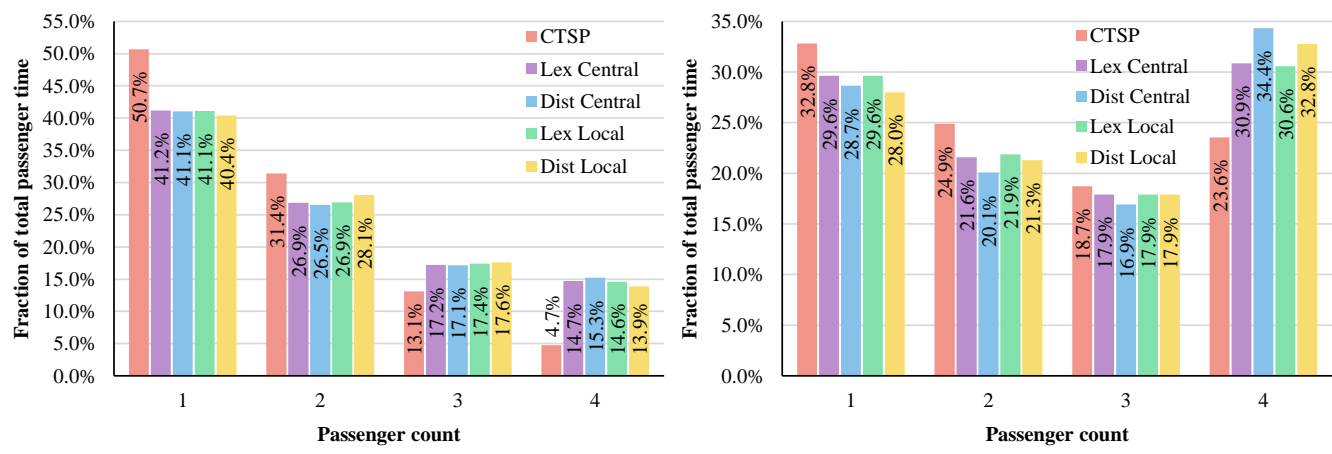

Figure 15: Fraction of Total Passenger Time Spent Serving Figure 16: Fraction of Total Passenger Time Spent Serving 1, 2, 3, and 4 Passengers for Clusters Inside City Limits. 1, 2, 3, and 4 Passengers for Clusters Outside City Limits.

configuration. Inside city limits, these fractions progressively decrease as the number of passengers increases. However the CTSPAV spends a larger fraction of its time serving three or four passengers than the CTSP, again reinforcing the advantage of the CTSPAV. This is due to two factors. First, mini routes in the CTSP must start and finish with the same driver. Second, the CTSP must also synchronize the inbound and outbound routes, since the same drivers are used for both. Because the schedules of the passengers very often differ, some of the mini routes cannot be used by the CTSP but can by the CTSPAV. This observation is carried over to clusters outside the city, whereby every configuration of the CTSPAV spends most of its time serving four passengers, while the same cannot be said for the CTSP. This observation can be attributed the longer transit phase of the routes for clusters outside the city combined with the vehicles being used to their full capacity during the transit phase by the CTSPAV.

In summary, the lexicographic objective for the CTSPAV has the greatest vehicle reduction potential, and the configuration of the depot does not appear to affect this potential. Its routes consistently cover the largest amount of trips on average, and they also have the longest passenger and busy durations, which are all desirable characteristics for an effective AV trip-sharing platform. The local depot configuration for the lexicographic objective does produce slightly better VMT results. This small benefit, however, may be outweighed by the logistical benefits of having a central depot, e.g., the convenience and cost effectiveness of having a central location for maintaining and refueling/recharging all AVs. The CTSPAV with the distance minimization objective and local depot configuration consistently produces the lowest VMT, however as mentioned earlier, the result is obtained at the expense of higher vehicle counts. Besides that, as shown in Figures 10 and 14 , the AVs also serve relatively fewer trips and spend more of their times being idle every day under this configuration.

\subsection{Cost Analysis}

This section reports a simple, coarse analysis to estimate the cost of operating the trip-sharing platform over a 5- and 10-year period. The analysis is not intended to be a sophisticated or complete measure of the total cost of the platform per se; instead, it is aimed to obtain a rough understanding of the trade-off between vehicle and operating costs of the platform. The analysis focuses only on the CTSPAV with the central depot configuration, as it is preferred over the local depot configuration due to its aforementioned logistical benefits. The analysis first considers vehicle-related costs (referred to simply as vehicle costs) and then operating costs. 

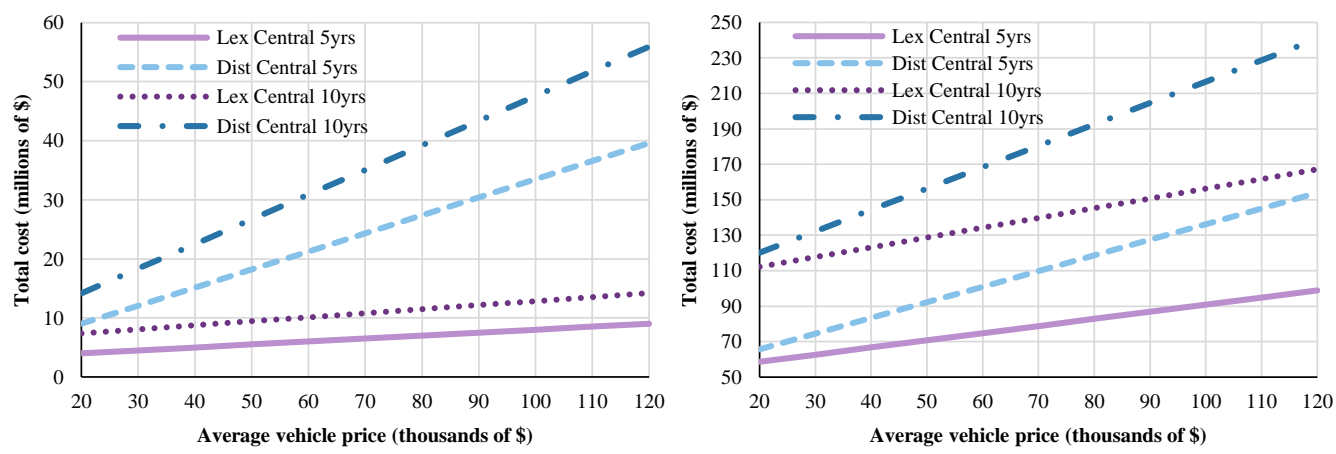

Figure 17: Total Vehicle Cost for CTSPAV Platform Inside Figure 18: Total Vehicle Cost for CTSPAV Platform OutCity Limits Over 5 and 10 Years. side City Limits Over 5 and 10 Years.

The vehicle cost is age-related and consists of the vehicle depreciation over $y$ years and a distance-related cost. This last cost consists of an average fuel cost of $\$ 0.15$ and an average vehicle-value depreciation of $\$ 0.08$ per mile traveled. An exponential decay function is used to model how a vehicle value depreciates with age. More precisely, its depreciation $\gamma$ over $y$ years is given by:

$$
\gamma=p-p(1-v)^{y}
$$

where $p$ is the vehicle's initial price and $v$ is its annual depreciation rate. This analysis uses a depreciation rate of $24 \%$ for the first year and $15 \%$ for subsequent years. The total vehicle cost over $y$ years is then obtained by multiplying the depreciation $\gamma$ of every vehicle over that period with the $\mathrm{VC}$ required to cover all daily trips, and then multiplying the distance cost with the average daily VMT over the time period considered, assuming trips are only made on weekdays.

Figures 17 and 18 show results of the vehicle cost analysis over 5- and 10-years for clusters inside and outside city limits respectively. In each figure, the horizontal axis displays the range of possible initial prices of an $\mathrm{AV}$, whereas the vertical axis displays the corresponding total vehicle cost. The figures show that, for the range of initial prices considered, the total vehicle cost is always dominated by the contributions from the vehicle-age cost. Therefore, the distanceminimization objective that requires larger VCs is always more expensive than the lexicographic objective, and this is true regardless of the location of the clusters or the time period considered for the analysis.

The operating cost considers the average annual cost of a parking permit for each vehicle combined with an estimated fixed cost for installing a charging station for each AV. For this analysis, an annual parking permit cost of $\$ 800$ is used together with a charging station installation cost of $\$ 1,400$ per vehicle. The total operating cost over $y$ years is then simply calculated by multiplying the charging station installation cost with the VC required to cover all daily trips, and then multiplying the annual parking permit cost with the $\mathrm{VC}$ and the number of years.

Figures 19 and 20 display results of the total operating cost as a function of the number of years for clusters inside and outside city limits respectively. The total parking cost of the CTSP and of the vehicles under no-sharing conditions are also included for additional perspective. Since there are no fixed costs associated with the CTSP or with the no-sharing conditions, their operating costs are lower than those of the CTSPAV in the beginning. However, as time increases, parking costs start to dominate, causing methods with larger VCs to be more expensive. In fact, 


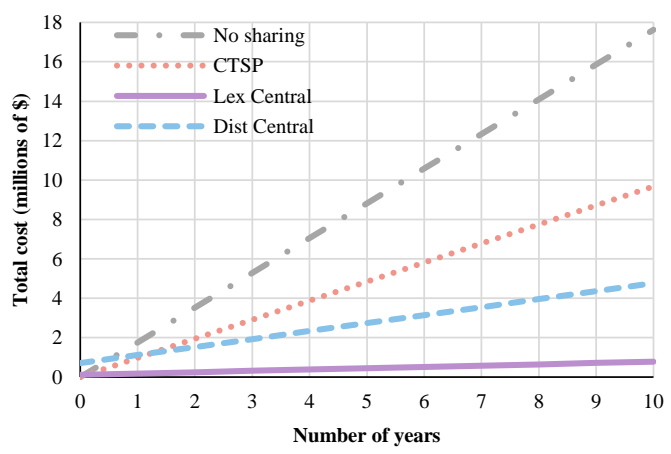

Figure 19: Total Operating Cost for CTSPAV Platform Inside City Limits Over 10 Years.

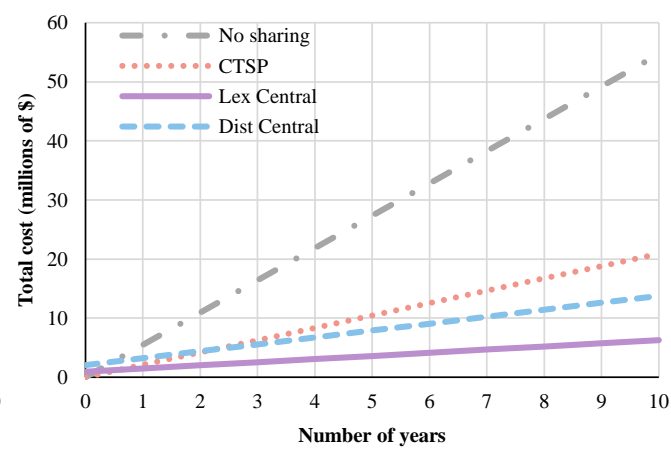

Figure 20: Total Operating Cost for CTSPAV Platform Outside City Limits Over 10 Years.

inside city limits, both CTSPAV methods considered become cheaper as early as the second year, while the same happens as early as the third year outside the city. The gap in operating costs between the different methods considered also increases with time and highlights the cost effectiveness of the CTSPAV with the lexicographic objective as it uses the fewest number of vehicles.

\subsection{Sensitivity to $\Delta$}

The parameter $\Delta$ represents the maximum amount of time by which each rider needs to shift (up or down) her desired arrival and departure times at a parking structure. It has a direct impact on the quality of service (QoS) of the riders, and it is desirable to have $\Delta$ be as small as possible. Limiting its value however restricts the flexibility of the schedules and could negatively impact trip shareability. To study the impact of varying $\Delta$ on the results of the CTSPAV, the procedure with the central depot configuration is applied to optimize ridesharing for every cluster with $\Delta$ set to $\{5,15\}$ mins. The results are then compared against those of $\Delta=10$ mins. Figures 21 and 22 compare aggregated VCs of all clusters inside and outside city limits respectively. Figures 23 and 24 then compare aggregated VMT from all clusters inside and outside the city respectively. Sensitivity results of the CTSP are also included in the figures, as well as the results under nosharing conditions and the percentage of each quantity as a fraction of the no-sharing results for additional perspective.

The results show that reducing $\Delta$ to 5 mins adversely affects the vehicle reduction capability of the CTSP, reducing its VC by approximately $12 \%$ inside the city (resp. $8 \%$ outside the city). Increasing $\Delta$ to 15 mins improves vehicle reduction by approximately $6 \%$ inside the city (resp. $4 \%$ outside the city). This is the evidence of a trade-off between QoS and trip shareability. In contrast, VC results of the CTSPAV with the lexicographic objective exhibit very little sensitivity to $\triangle$, whereby the VCs change by $\leq 1 \%$ as $\Delta$ is varied by \pm 5 mins. This bodes very well for the CTSPAV as it indicates that a reduction in $\Delta$ to improve the quality of service for the riders will not have a significant impact on its vehicle reduction capability. Finally, the VCs of the CTSPAV with the distance-minimization objective display a modest sensitivity to $\Delta$, whereby decreasing $\Delta$ by 5 mins degrades VCs by approximately 5\% inside the city (resp. 3\% outside the city), and increasing it by 5 mins improves VCs by approximately $2 \%$ insde the city (resp. $1 \%$ outside the city). 


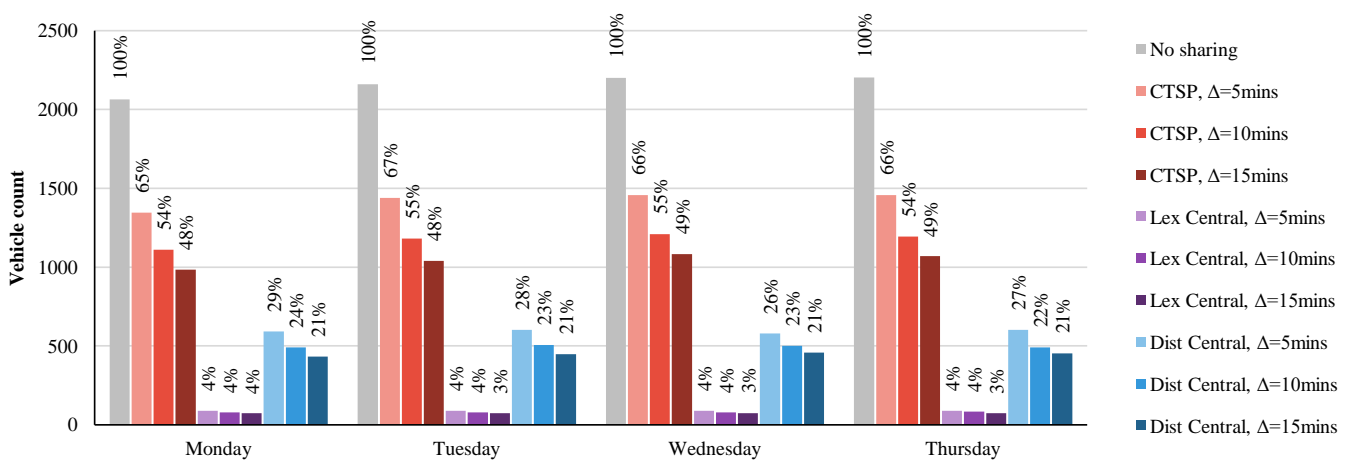

Figure 21: Aggregate Vehicle Count Results Inside City Limits for $\Delta=\{5,10,15\}$ mins.

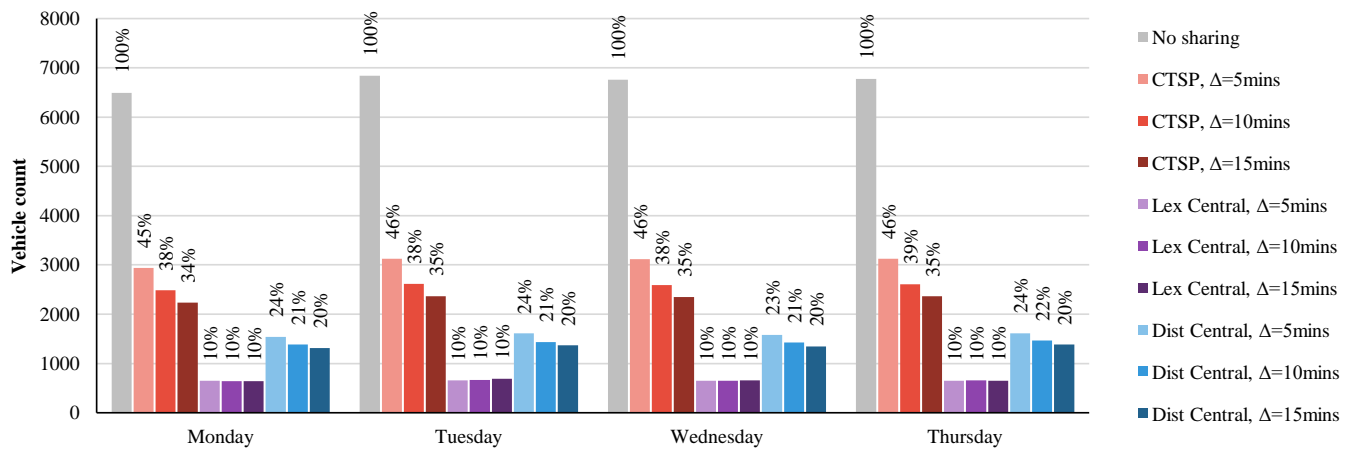

Figure 22: Aggregate Vehicle Count Results Outside City Limits for $\Delta=\{5,10,15\}$ mins.

The sensitivity analysis on aggregated VMT paints a different picture, whereby every method considered exhibits comparable sensitivity to the variations in $\Delta$. The results once again display a trade-off, this time between QoS and travel distance reduction, evident from the latter decreasing as $\Delta$ is increased and vice versa. The increase in VMT of the CTSPAV, regardless of objective function or position of clusters, when $\Delta$ is reduced stems from the reduction of opportunities for trip aggregation as a result of the tighter time windows. The AVs would therefore need to increase back-and-forth traveling between the parking structures and neighborhoods to serve the same amount of trips, leading to corresponding increases in their travel distance.

\subsection{Sensitivity to $R$}

The parameter $R$ is yet another parameter that may influence the QoS of the riders, as it directly influences the maximum amount of time every rider spends in a vehicle. Limiting the value of $R$ improves QoS as it leads to shorter ride durations. However, it also results in less flexible trip schedules, which could consequently reduce the potential for trip aggregation. Therefore, one would anticipate a trade-off between QoS and trip shareability when varying $R$ similar to that observed in the sensitivity analysis on $\Delta$. To investigate this trade-off, a sensitivity analysis is conducted by setting $R=\{25 \%, 75 \%\}$, applying the procedure with the central depot configuration to optimize ridesharing for every cluster, and comparing the results with those of $R=50 \%$. 


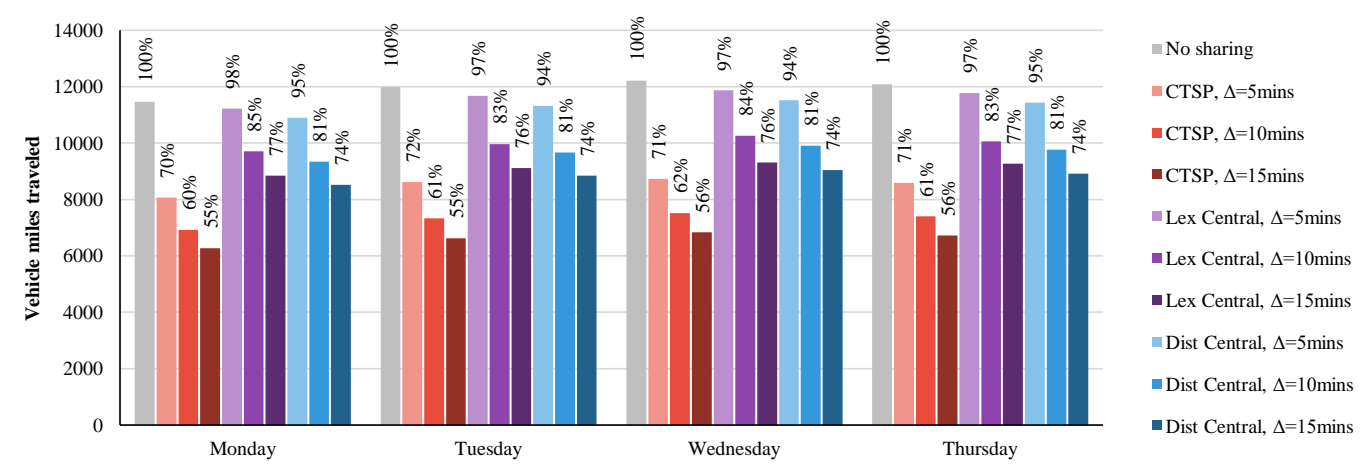

Figure 23: Aggregate Vehicle Miles Traveled Inside City Limits for $\Delta=\{5,10,15\}$ mins.

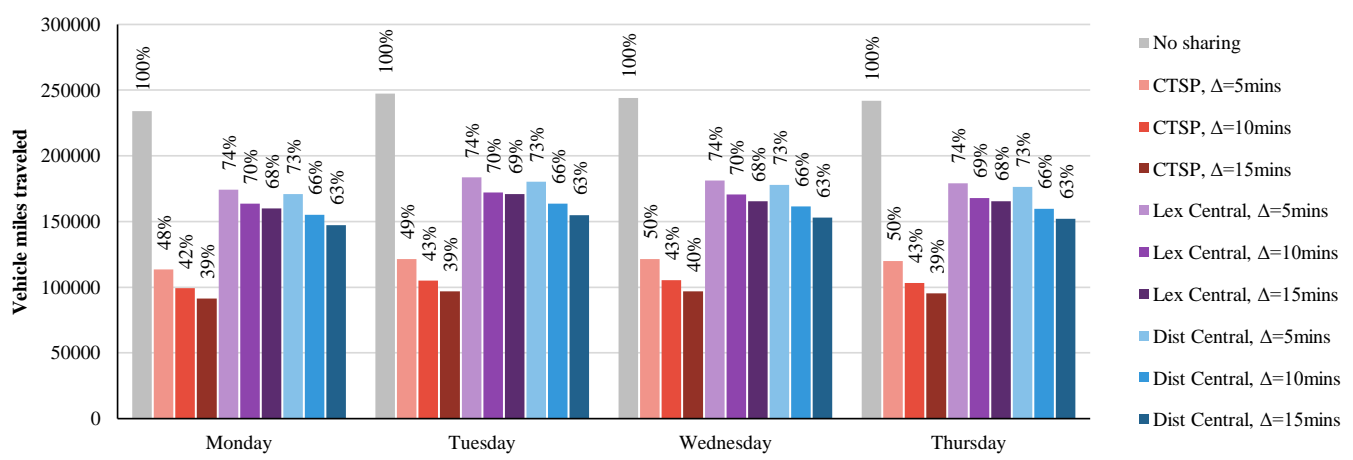

Figure 24: Aggregate Vehicle Miles Traveled Outside City Limits for $\Delta=\{5,10,15\}$ mins.

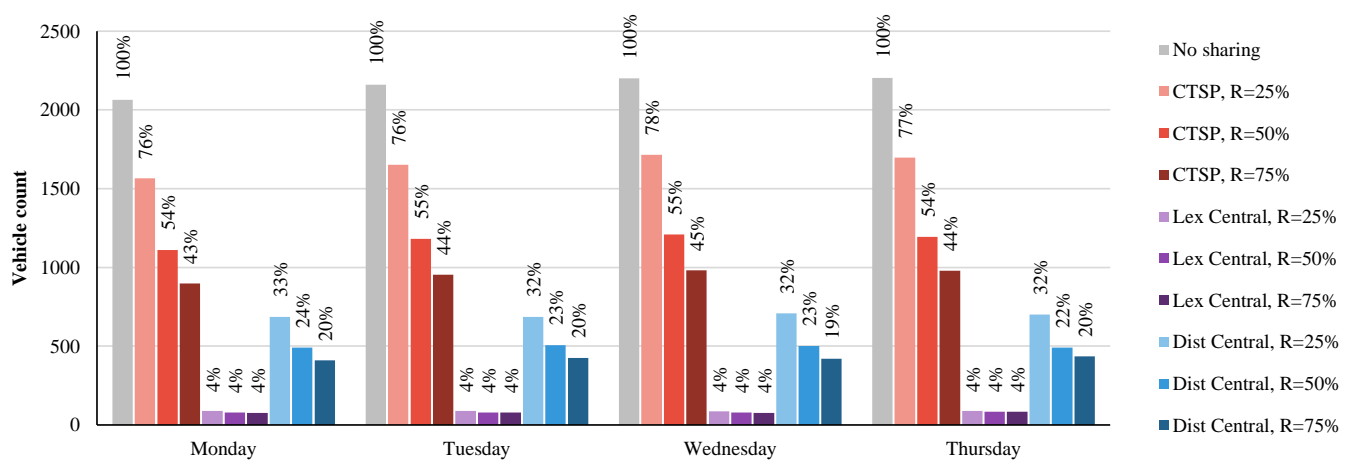

Figure 25: Aggregate Vehicle Count Results Inside City Limits for $R=\{25 \%, 50 \%, 75 \%\}$.

Results of the analysis on VCs inside and outside city limits are summarized in Figures 25 and 26 respectively, whereas the analysis on VMT inside and outside city limits are displayed in Figures 27 and 28 respectively. Similar to previous analyses, results from the original CTSP, from the no-sharing condition, and percentages of each quantity as a fraction of the no-sharing results are included for reference.

The VC results of the CTSP display the expected trade-off described earlier where they ap- 


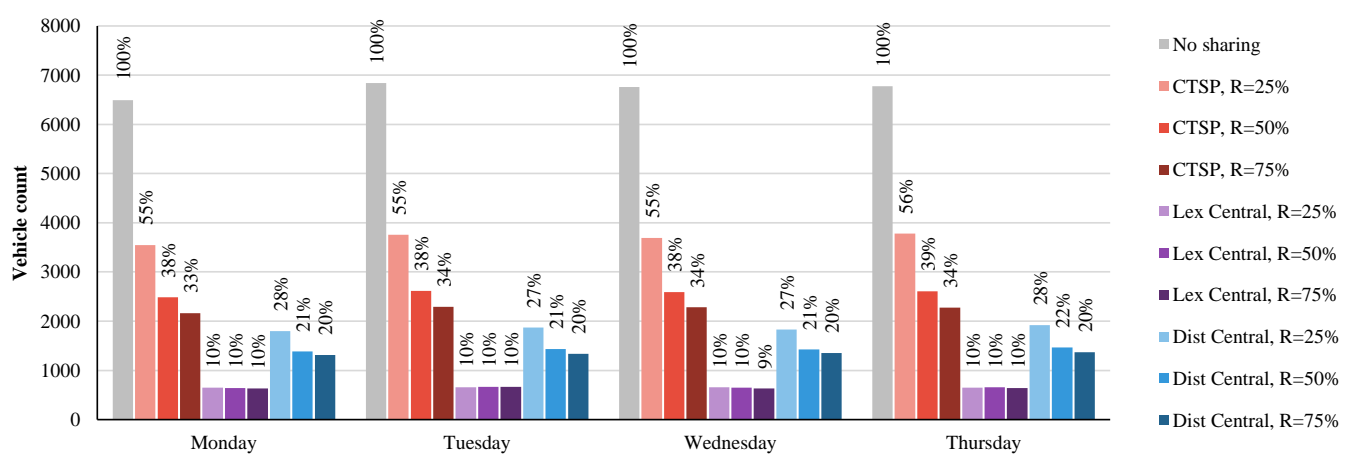

Figure 26: Aggregate Vehicle Count Results Outside City Limits for $R=\{25 \%, 50 \%, 75 \%\}$.

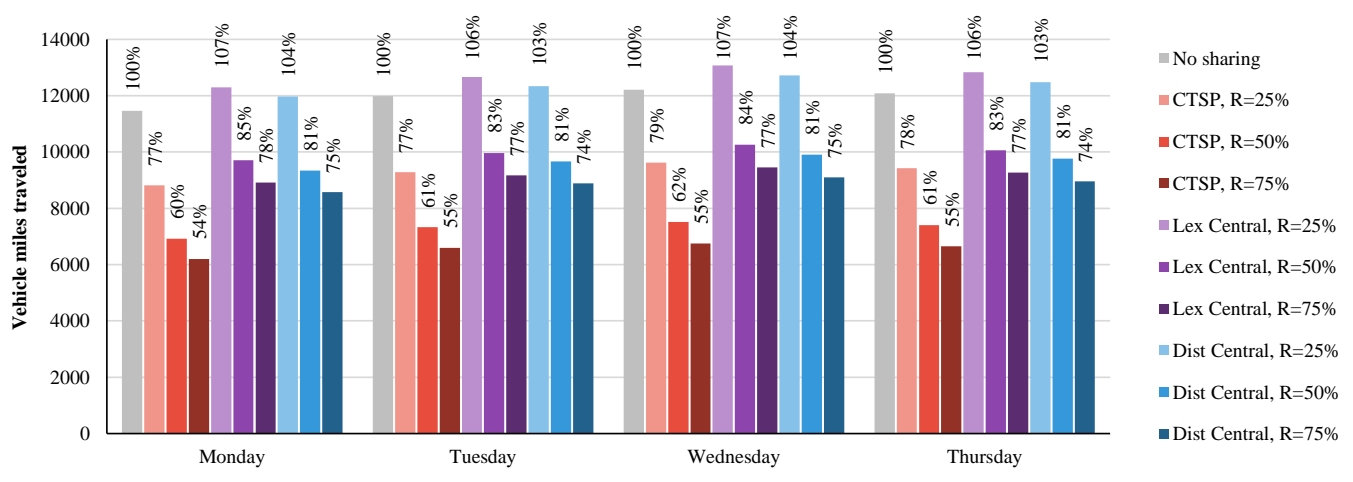

Figure 27: Aggregate Vehicle Miles Traveled Inside City Limits for $R=\{25 \%, 50 \%, 75 \%\}$.

pear to be very sensitive to variations in $R$. Vehicle reduction significantly degrades by approximately $22 \%$ inside the city (resp. $17 \%$ outside the city) when $R$ is reduced to $25 \%$, and it improves by approximately $11 \%$ inside the city (resp. 5\% outside the city) when $R$ is increased to $75 \%$. This indicates that attempts to reduce the maximum ride durations will result in significantly reduced trip shareability in the CTSP. In contrast, such a drawback is not evident from the results of the CTSPAV with the lexicographic objective, as its VCs exhibit very little sensitivity to changes in $R$. The results show changes that are $<1 \%$ both inside and outside the city as $R$ is varied by $\pm 25 \%$ This result provides another positive outlook for the CTSPAV as it promises that the vehicle reductions will not be adversely affected by attempts to increase QoS by reducing the maximum ride durations of the riders. Finally, the VC results of the CTSPAV with the distance minimization objective displays a relatively modest sensitivity to $R$, whereby decreasing $R$ to $25 \%$ increased VCs by approximately $9 \%$ inside the city (resp. $6 \%$ outside the city), and increasing $R$ to $75 \%$ reduced VCs by approximately $3 \%$ inside the city (resp. $1 \%$ outside the city).

Analysis of the VMT results reveals different observations. Inside city limits, both objective

\footnotetext{
${ }^{2}$ For the clusters outside city limits, the column-generation phase generated on average $34 \%$ more columns for $R=$ $75 \%$ than it did for $R=50 \%$, which caused the MIP to be significantly harder to solve. The MIP time limit for these instances is therefore extended to $2 \mathrm{hrs}$ to account for this increase in complexity.
} 


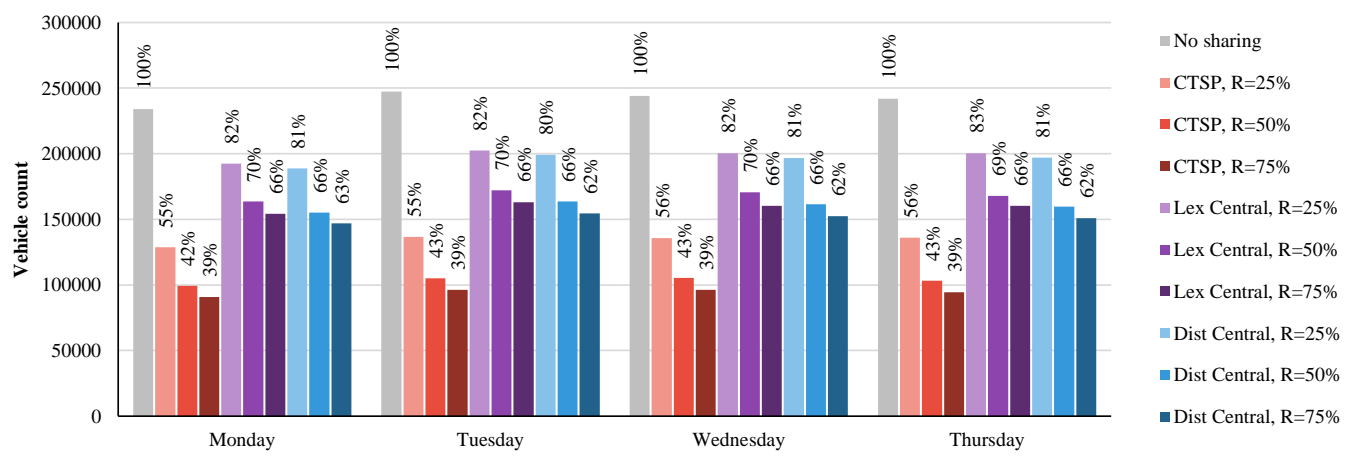

Figure 28: Aggregate Vehicle Miles Traveled Outside City Limits for $R=\{25 \%, 50 \%, 75 \%\}$.

functions of the CTSPAV appear to be more sensitive to changes in $R$ than the CTSP; decreasing $R$ to $25 \%$ leads to approximately a $23 \%$ increase in VMT for both versions of the CTSPAV (compared to a $17 \%$ increase for the CTSP). In fact, the increase is so significant in this case that their aggregated values exceed those for the no-sharing condition. This indicates that the opportunities for trip aggregation in this case is diminished to the point that any savings in travel distance is overshadowed by a more significant increase in back-and-forth traveling resulting from having to cover the trips with approximately the same amount of vehicles. Outside city limits, the VMT of every method considered exhibit comparable sensitivity to changes in $R$, once again displaying a trade-off between QoS and travel distance reduction.

\subsection{Effect of Increasing Vehicle Capacity}

This section explores the effects of increasing vehicle capacity, $K$, on the results of the CTSPAV to investigate if there are any benefits to be gained from using vehicles with larger capacity. The results are obtained by varying $K$ between 1 to 8 and applying the CTSPAV procedure with the central depot configuration on all trips made on the Wednesday of week $23^{3}$ Figure 29 shows the aggregated vehicle counts from all clusters for every $K$ value, while Figure 30 does the same for the aggregated vehicle miles traveled. The percentages in both figures indicate each quantity as a fraction of the no-sharing values.

Both figures reveal that marginal improvements (reductions) in VC and VMT decrease with increasing $K$. In fact, they show that almost no improvement is obtained for both VC and VMT beyond $K=5$, and the biggest improvement is obtained when increasing $K$ from 1 to 2 . These results are consistent with the findings from Farhan and Chen (2018) which focused on an SAV system for on-demand trips, whereby they discovered that the decreases to the fleet size was marginal for $K>2$. It must also be noted that the marginal benefits obtained from increasing $K$ is accompanied by an exponential increase in problem complexity (in fact, the very slight increase in VC and VMT for $K>6$ can be attributed the increase in problem complexity and

\footnotetext{
${ }^{3}$ To accommodate the exponential increase in problem complexity that results from the increase in $K$, the following changes were applied to the CTSPAV procedure for $K>4$. A 10-minutes timeout is applied to the label-setting dynamic

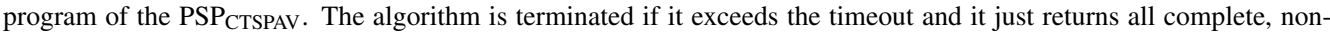
dominated mini routes discovered at that point with negative reduced costs. The column-generation procedure is also seeded all with feasible mini routes covering up to 2 trips which are obtained from an exhaustive search procedure Finally, the time budget for the column-generation phase is extended to 2 hours.
} 

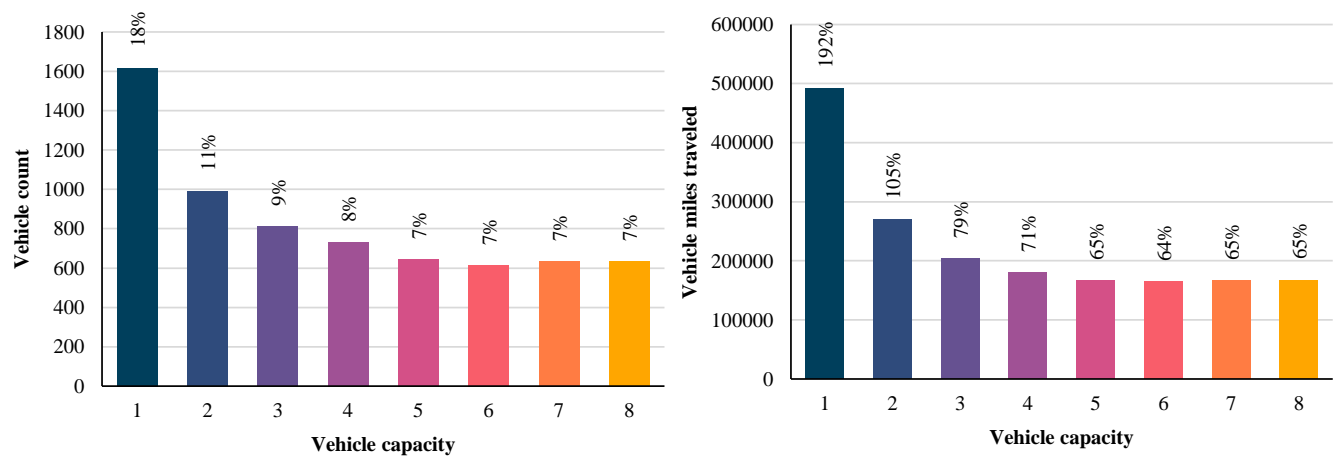

Figure 29: Effect of Increasing Vehicle Capacity on Aggre- Figure 30: Effect of Increasing Vehicle Capacity on Aggregated Vehicle Count. gated Vehicle Miles Traveled.

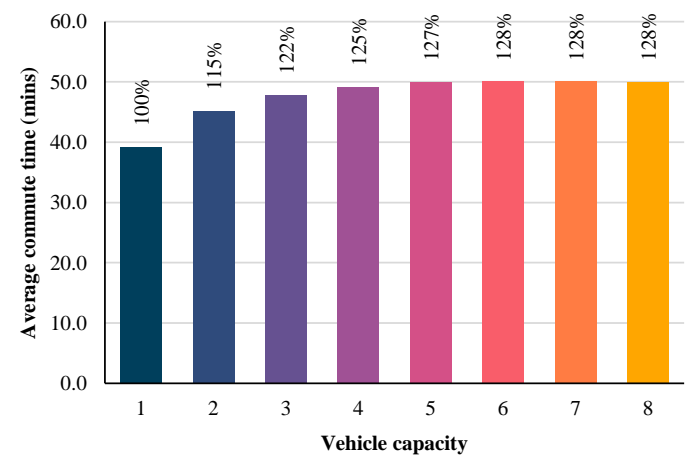

Figure 31: Effect of Increasing Vehicle Capacity on Average Commute Time.

the procedure not being able to find better solutions even with the extended time budget). The general trend of diminishing marginal benefits can probably be attributed to the unavailability of more trips with compatible itineraries that are needed to fully utilize the larger vehicle capacities from the problem instances considered.

Finally, Figure 31 shows the effect of increasing $K$ on the average ride duration per commuter. The percentages represent each quantity as a fraction of the value when $K=1$. Unsurprisingly, the best average commute time is obtained when $K=1$ as the rides are not shared under this setting, and the times increase with increasing $K$ due to the corresponding increase in ridesharing. The diminishing nature of the marginal increases in average commute time as $K$ is increased also appears to mirror the marginal decreases in VC or VMT. The figure also shows that when $K=4$, the average commute time only increases by $25 \%$ (even though $R=0.50$ ), which bodes very well for maintaining the QoS of riders of the CTSPAV.

\section{Conclusion}

This work originated from the desire to understand the potential benefits of autonomous vehicles on a car-pooling platform that maximizes ridesharing for the commuting trips of a community. The central problem powering the platform is the CTSP: it was originally conceived by 
Hasan et al. (2018) to reduce parking utilization and traffic congestion in urban areas by leveraging the structure of commuting patterns and urban communities. The CTSP was shown to reduce the number of vehicles significantly on a real case study.

Given that vehicles are idle for most of the day by definition of car-pooling, it is interesting to study how much additional reduction in fleet size would come from using autonomous vehicles, as well as the impact on miles traveled. To answer that question, this paper defined the CTSPAV problem and proposed two column-generation procedures to obtain high-quality solutions. The first approach (the CTSPAV procedure) assembles feasible mini routes into an overall routing plan, while the second approach (the DARP procedure) reduces the CTSPAV into a DARP. The optimization problems considered (1) a lexicographic objective that first minimizes the required vehicle count and then their total distance, and (2) a distance minimization objective that just minimizes the total travel distance.

The CTSPAV was evaluated on a large-scale, real-world dataset of commute trips from the city of Ann Arbor, Michigan, which contains detailed information for an average of 9,000 daily trips over a month. The experimental results revealed that the CTSPAV procedure with the lexicographic objective reduces daily vehicle usage by $92 \%$ while at the same time also reducing vehicle miles traveled by $30 \%$. Its vehicle reduction results represent a $34 \%$ improvement relative to that of the CTSP. Examining the solutions show that the CTSPAV generates significantly longer routes by traveling back and forth between the communities and the commuting destination (and vice versa). A cost analysis also showed that fleets of AV vehicles are eminently viable from an economic standpoint in this setting. Finally, sensitivity analyses revealed that the vehicle count results of the lexicographic objective are more resilient to variations in the size of the time windows and the length of the ride-duration limits of the trips. The number of vehicles required to cover all trips changed by less than $1 \%$ as the two parameters are varied, while the same cannot be said about the CTSP.

\section{Acknowledgement}

We would like to thank Stephen Dolen from Logistics, Transportation, and Parking of the University of Michigan for his assistance in obtaining the dataset used in this research. Part of this research was funded by the Rackham Graduate Student Research Grant, computational resources and services provided by Advanced Research Computing at the University of Michigan, and NSF Leap HI proposal NSF-1854684.

\section{References}

Agatz, N., Erera, A., Savelsbergh, M., Wang, X., 2012. Optimization for dynamic ride-sharing: A review. European Journal of Operational Research 223, 295 - 303. URL: http://www.sciencedirect.com/science/article/ pii/S0377221712003864 doi https://doi.org/10.1016/j.ejor.2012.05.028

Agatz, N.A., Erera, A.L., Savelsbergh, M.W., Wang, X., 2011. Dynamic ride-sharing: A simulation study in metro atlanta. Transportation Research Part B: Methodological 45, 1450 - 1464. URL: http://www. sciencedirect.com/ science/article/pii/S0191261511000671 doi https://doi.org/10.1016/j.trb.2011.05.017 select Papers from the 19th ISTTT.

Alazzawi, S., Hummel, M., Kordt, P., Sickenberger, T., Wieseotte, C., Wohak, O., 2018. Simulating the impact of

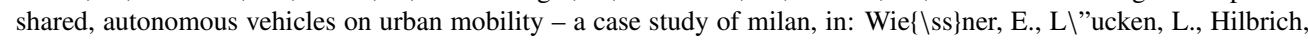
R., Fl\"otter\"od, Y.P., Erdmann, J., Bieker-Walz, L., Behrisch, M. (Eds.), SUMO 2018- Simulating Autonomous and Intermodal Transport Systems, EasyChair. pp. 94-110. URL: https://easychair.org/publications/paper/ 28wc doi $10.29007 / 2 \mathrm{n} 4 \mathrm{~h}$ 
Alonso-Mora, J., Samaranayake, S., Wallar, A., Frazzoli, E., Rus, D., 2017. On-demand high-capacity ride-sharing via dynamic trip-vehicle assignment. Proceedings of the National Academy of Sciences 114, 462-467. URL: https://www.pnas.org/content/114/3/462 doi 10.1073/pnas.1611675114 arXiv:https://www.pnas.org/content/114/3/462.full.pdf

Baldacci, R., Maniezzo, V., Mingozzi, A., 2004. An exact method for the car pooling problem based on lagrangean column generation. Operations Research 52, 422-439. URL: https://doi.org/10.1287/opre.1030.0106 doi 10.1287/opre.1030.0106 arXiv:https://doi.org/10.1287/opre.1030.0106

Bard, J.F., Kontoravdis, G., Yu, G., 2002. A branch-and-cut procedure for the vehicle routing problem with time windows. Transportation Science 36, 250-269. URL: https://doi.org/10.1287/trsc.36.2.250.565 doi 10.1287/ trsc.36.2.250.565 arXiv:https://doi.org/10.1287/trsc.36.2.250.565

Beasley, J.E., Christofides, N., 1989. An algorithm for the resource constrained shortest path problem. Networks 19, 379394. URL: https://onlinelibrary.wiley.com/doi/abs/10.1002/net.3230190402 doi 10.1002/net. 3230190402 arXiv:https://onlinelibrary.wiley.com/doi/pdf/10.1002/net.3230190402

Bodin, L., Sexton, T., 1986. The multi-vehicle subscriber dial-a-ride problem. TIMS Studies in Management Science 22, 73-86.

Boland, N., Dethridge, J., Dumitrescu, I., 2006. Accelerated label setting algorithms for the elementary resource constrained shortest path problem. Operations Research Letters 34, 58 - 68. URL: http://www. sciencedirect . com/science/article/pii/S0167637705000040 doi https://doi.org/10.1016/j.orl.2004.11.011

Bongiovanni, C., Kaspi, M., Geroliminis, N., 2019. The electric autonomous dial-a-ride problem. Transportation Research Part B: Methodological 122, 436 - 456. URL: http://www.sciencedirect.com/science/article/ pii/S0191261517309669 doi https://doi.org/10.1016/j.trb.2019.03.004

Borndörfer, R., Grötschel, M., Löbel, A., 2001. Scheduling duties by adaptive column generation. ZIB-Report 01-02 Konrad-Zuse-Zentrum für Informationstechnik Berlin.

Bräysy, O., Gendreau, M., 2005. Vehicle routing problem with time windows, part ii: Metaheuristics. Transportation Science 39, 119-139. URL: https://pubsonline.informs.org/doi/abs/10.1287/trsc.1030.0057 doi 10. 1287/trsc.1030.0057 arXiv:https://pubsonline.informs.org/doi/pdf/10.1287/trsc.1030.0057

Chabrier, A., 2006. Vehicle routing problem with elementary shortest path based column generation. Comput. Oper. Res 33, 2972-2990. URL:https://doi.org/10.1016/j.cor.2005.02.029 doi 10.1016/j.cor.2005.02.029

Cordeau, J.F., 2006. A branch-and-cut algorithm for the dial-a-ride problem. Operations Research 54 573-586. URL: https://doi.org/10.1287/opre.1060.0283 doi 10.1287/opre.1060.0283 arXiv:https://doi.org/10.1287/opre.1060.0283

Cordeau, J.F., Desaulniers, G., Desrosiers, J., Solomon, M.M., Soumis, F., 2002. VRP with time windows, in: Toth, P., Vigo, D. (Eds.), The Vehicle Routing Problem. SIAM monographs on discrete mathematics and applications, Philadelphia, PA, USA. chapter 7, pp. 157-193. URL: https: //epubs.siam.org/doi/abs/10.1137/1.9780898718515.ch7 doi 10.1137/1.9780898718515.ch7 arXiv:https://epubs.siam.org/doi/pdf/10.1137/1.9780898718515.ch7

Cordeau, J.F., Laporte, G., 2003a. The dial-a-ride problem (darp): Variants, modeling issues and algorithms. Quarterly Journal of the Belgian, French and Italian Operations Research Societies 1, 89-101. URL: https://doi.org/10. 1007/s10288-002-0009-8 doi 10.1007/s10288-002-0009-8

Cordeau, J.F., Laporte, G., 2003b. A tabu search heuristic for the static multi-vehicle dial-a-ride problem. Transportation Research Part B: Methodological 37, 579 - 594. URL: http://www.sciencedirect.com/science/article/ pii/S0191261502000450 doi https://doi.org/10.1016/S0191-2615(02)00045-0

Cordeau, J.F., Laporte, G., 2007. The dial-a-ride problem: models and algorithms. Annals of Operations Research 153 , 29-46. URL: https://doi.org/10.1007/s10479-007-0170-8 doi 10.1007/s10479-007-0170-8

Dantzig, G.B., Wolfe, P., 1960. Decomposition principle for linear programs. Operations Research 8, 101-111. URL: https://doi.org/10.1287/opre.8.1.101 doi 10.1287/opre.8.1.101 arXiv:https://doi.org/10.1287/opre.8.1.101

Desrochers, M., 1988. An algorithm for the shortest path problem with resource constraints. Technical Report G-88-27. Les Cahiers du GERAD. Montreal (Quebec), Canada.

Desrochers, M., Desrosiers, J., Solomon, M., 1992. A new optimization algorithm for the vehicle routing problem with time windows. Operations Research 40, 342-354. URL: https://doi.org/10.1287/opre.40.2.342 doi 10.1287/opre.40.2.342 arXiv:https://doi.org/10.1287/opre.40.2.342

Desrochers, M., Soumis, F., 1988. A generalized permanent labelling algorithm for the shortest path problem with time windows. INFOR: Information Systems and Operational Research 26, 191-212. URL: https://doi.org/10.1080/03155986.1988.11732063. arXiv:https://doi.org/10.1080/03155986.1988.11732063

Desrosiers, J., Pelletier, P., Soumis, F., 1983. Plus court chemin avec contraintes d'horaires. RAIRO - Operations Research - Recherche Opérationnelle 17, 357-377. URL: http://eudml .org/doc/104840

Desrosiers, J., Soumis, F., Desrochers, M., 1984. Routing with time windows by column generation. Networks $14,545-$ 
565. URL: https://onlinelibrary.wiley.com/doi/abs/10.1002/net.3230140406 doi 10.1002/net. 3230140406 arXiv:https://onlinelibrary.wiley.com/doi/pdf/10.1002/net.3230140406

Dia, H., Javanshour, F., 2017. Autonomous shared mobility-on-demand: Melbourne pilot simulation study. Transportation Research Procedia 22, 285 - 296. URL: http://www.sciencedirect.com/science/article/pii/ S2352146517301709 doi https://doi.org/10.1016/j.trpro.2017.03.035 19th EURO Working Group on Transportation Meeting, EWGT2016, 5-7 September 2016, Istanbul, Turkey.

Drexl, M., 2013. A note on the separation of subtour elimination constraints in elementary shortest path problems. European Journal of Operational Research 229, 595 - 598. URL: http://www.sciencedirect.com/science/ article/pii/S0377221713002178 doi https://doi.org/10.1016/j.ejor.2013.03.009

Dror, M., 1994. Note on the complexity of the shortest path models for column generation in vrptw. Operations Research 42, 977-978. URL: https://doi.org/10.1287/opre.42.5.977. doi 10.1287/opre.42.5.977 arXiv:https://doi.org/10.1287/opre.42.5.977

Dumas, Y., Desrosiers, J., Soumis, F., 1991. The pickup and delivery problem with time windows. European Journal of Operational Research 54, 7 - 22. URL: http://www.sciencedirect.com/science/article/pii/ 037722179190319Q doi https://doi.org/10.1016/0377-2217(91)90319-Q

Farhan, J., Chen, T.D., 2018. Impact of ridesharing on operational efficiency of shared autonomous electric vehicle fleet. Transportation Research Part C: Emerging Technologies 93, 310 - 321. URL: http://www. sciencedirect.com/ science/article/pii/S0968090X18305758 doi/https://doi.org/10.1016/j.trc.2018.04.022

Farley, A.A., 1990. A note on bounding a class of linear programming problems, including cutting stock problems. Operations Research 38, 922-923. URL: https://doi.org/10.1287/opre.38.5.922 doi 10.1287/opre.38. 5.922 arXiv:https://doi.org/10.1287/opre.38.5.922

Feillet, D., Dejax, P., Gendreau, M., Gueguen, C., 2004. An exact algorithm for the elementary shortest path problem with resource constraints: Application to some vehicle routing problems. Networks 44, 216229. URL: https://onlinelibrary.wiley.com/doi/abs/10.1002/net.20033 doi 10.1002/net.20033 arXiv:https://onlinelibrary.wiley.com/doi/pdf/10.1002/net.20033

Firat, M., Woeginger, G.J., 2011. Analysis of the dial-a-ride problem of hunsaker and savelsbergh. Operations Research Letters 39, 32 - 35. URL: http://www.sciencedirect.com/science/article/pii/S0167637710001495 doi https://doi.org/10.1016/j.orl.2010.11.004

Friedrich, B., 2015. Verkehrliche wirkung autonomer fahrzeuge, in: Maurer, M., Gerdes, J.C., Lenz, B., Winner, H. (Eds.), Autonomes Fahren: Technische, rechtliche und gesellschaftliche Aspekte. Springer Berlin Heidelberg, Berlin, Heidelberg, pp. 331-350. URL: https://doi.org/10.1007/978-3-662-45854-9_16 doi 10.1007/ 978-3-662-45854-9_16

Gschwind, T., Irnich, S., 2015. Effective handling of dynamic time windows and its application to solving the dial-a-ride problem. Transportation Science 49, 335-354. URL: https://doi.org/10.1287/trsc.2014.0531 doi 10 . 1287/trsc.2014.0531 arXiv:https://doi.org/10.1287/trsc.2014.0531

Gurumurthy, K.M., Kockelman, K.M., 2018. Analyzing the dynamic ride-sharing potential for shared autonomous vehicle fleets using cellphone data from orlando, florida. Computers, Environment and Urban Systems $71,177-$ 185. URL: http://www.sciencedirect.com/science/article/pii/S0198971517305732 doi https:// doi.org/10.1016/j.compenvurbsys.2018.05.008

Hasan, M.H., Van Hentenryck, P., 2020. The flexible and real-time commute trip sharing problems. Constraints URL: https://doi.org/10.1007/s10601-020-09310-5 doi 10.1007/s10601-020-09310-5

Hasan, M.H., Van Hentenryck, P., Budak, C., Chen, J., Chaudhry, C., 2018. Community-based trip sharing for urban commuting, in: McIlraith, S., Weinberger, K. (Eds.), Proceedings of the Thirty-Second AAAI Conference on Artificial Intelligence, AAAI Press, Palo Alto, California, USA. pp. 6589-6597.

Hasan, M.H., Van Hentenryck, P., Legrain, A., 2020. The commute trip-sharing problem. Transportation Science URL: https://doi.org/10.1287/trsc.2019.0969 doi 10.1287/trsc.2019.0969 arXiv:https://doi.org/10.1287/trsc.2019.0969

Haugland, D., Ho, S.C., 2010. Feasibility testing for dial-a-ride problems, in: Chen, B. (Ed.), Algorithmic Aspects in Information and Management, Springer Berlin Heidelberg, Berlin, Heidelberg. pp. 170-179.

Hunsaker, B., Savelsbergh, M., 2002. Efficient feasibility testing for dial-a-ride problems. Operations Research Letters 30, 169 - 173. URL: http://www.sciencedirect.com/science/article/pii/S0167637702001207 doi https://doi .org/10.1016/S0167-6377(02)00120-7

Hyland, M., Mahmassani, H.S., 2018. Dynamic autonomous vehicle fleet operations: Optimization-based strategies to assign avs to immediate traveler demand requests. Transportation Research Part C: Emerging Technologies 92, 278 - 297. URL: http://www.sciencedirect.com/science/article/pii/S0968090X18306028 doi https:// doi.org/10.1016/j.trc.2018.05.003

Hyland, M.F., Mahmassani, H.S., 2017. Taxonomy of shared autonomous vehicle fleet management problems to inform future transportation mobility. Transportation Research Record 2653, 26-34. URL: https://doi.org/10.3141/ 2653-04 doi 10.3141/2653-04 arXiv:https://doi.org/10.3141/2653-04 
Irnich, S., Desaulniers, G., 2005. Shortest path problems with resource constraints, in: Desaulniers, G., Desrosiers, J., Solomon, M.M. (Eds.), Column Generation. Springer US, Boston, MA, pp. 33-65. URL: https://doi.org/10 . 1007/0-387-25486-2_2 doi 10.1007/0-387-25486-2_2

Irnich, S., Villeneuve, D., 2006. The shortest-path problem with resource constraints and $k$-cycle elimination for $k \geq 3$. INFORMS Journal on Computing 18, 391-406. URL: https://doi.org/10.1287/ijoc.1040.0117. doi 10. 1287/ijoc.1040.0117 arXiv:https://doi.org/10.1287/ijoc.1040.0117

Jaw, J.J., Odoni, A.R., Psaraftis, H.N., Wilson, N.H., 1986. A heuristic algorithm for the multi-vehicle advance request dial-a-ride problem with time windows. Transportation Research Part B: Methodological 20, 243 - 257. URL: http://www.sciencedirect.com/science/article/pii/0191261586900202 doi https://doi.org/10. 1016/0191-2615(86)90020-2

Kallehauge, B., Boland, N., Madsen, O.B., 2007. Path inequalities for the vehicle routing problem with time windows. Networks 49, 273-293. URL: https://onlinelibrary.wiley.com/doi/abs/10.1002/net.20178 doi 10. 1002/net.20178 arXiv:https://onlinelibrary.wiley.com/doi/pdf/10.1002/net.20178

Kallehauge, B., Larsen, J., Madsen, O.B., 2006. Lagrangian duality applied to the vehicle routing problem with time windows. Computers \& Operations Research 33, 1464 - 1487. URL: http://www.sciencedirect.com/science/ article/pii/S0305054804003028 doi/https://doi.org/10.1016/j.cor.2004.11.002

Kohl, N., Desrosiers, J., Madsen, O.B.G., Solomon, M.M., Soumis, F., 1999. 2-path cuts for the vehicle routing problem with time windows. Transportation Science 33, 101-116. URL: https://doi.org/10.1287/trsc.33.1.101 doi $10.1287 / \operatorname{trsc} .33 .1 .101$ arXiv: https://doi.org/10.1287/trsc.33.1.101

Kohl, N., Madsen, O.B.G., 1997. An optimization algorithm for the vehicle routing problem with time windows based on lagrangian relaxation. Operations Research 45, 395-406. URL: https://doi .org/10.1287/opre.45.3.395 doi 10.1287/opre.45.3.395 arXiv:https://doi.org/10.1287/opre.45.3.395

Lasdon, L.S., 1970. Optimization theory for large systems. MacMillan.

Lübbecke, M.E., Desrosiers, J., 2005. Selected topics in column generation. Operations Research 53, 1007-1023. URL: https://doi.org/10.1287/opre.1050.0234 doi 10.1287/opre.1050.0234 arXiv:https://doi.org/10.1287/opre.1050.0234

Ma, J., Li, X., Zhou, F., Hao, W., 2017. Designing optimal autonomous vehicle sharing and reservation systems: A linear programming approach. Transportation Research Part C: Emerging Technologies 84, $124-$ 141. URL: http://www.sciencedirect.com/science/article/pii/S0968090X17302322 doi https:// doi.org/10.1016/j.trc.2017.08.022

Martinez, L.M., Viegas, J.M., 2017. Assessing the impacts of deploying a shared self-driving urban mobility system: An agent-based model applied to the city of lisbon, portugal. International Journal of Transportation Science and Technology 6, 13 - 27. URL: http://www.sciencedirect.com/science/article/pii/S2046043016300442 doi https://doi.org/10.1016/j.ijtst.2017.05.005 connected and Automated Vehicles: Effects on Traffic, Mobility and Urban Design.

Mena-Oreja, J., Gozalvez, J., Sepulcre, M., 2018. Effect of the configuration of platooning maneuvers on the traffic flow under mixed traffic scenarios, in: 2018 IEEE Vehicular Networking Conference (VNC), pp. 1-4. doi 10.1109/VNC. 2018.8628381

Milakis, D., van Arem, B., van Wee, B., 2017. Policy and society related implications of automated driving: A review of literature and directions for future research. Journal of Intelligent Transportation Systems 21, 324348. URL: https://doi.org/10.1080/15472450.2017.1291351 doi 10.1080/15472450.2017.1291351 arXiv:https://doi.org/10.1080/15472450.2017.1291351

Mourad, A., Puchinger, J., Chu, C., 2019. A survey of models and algorithms for optimizing shared mobility. Transportation Research Part B: Methodological 123, 323 - 346. URL: http://www.sciencedirect.com/science/ article/pii/S0191261518304776 doi/https://doi.org/10.1016/j.trb.2019.02.003

Narayanan, S., Chaniotakis, E., Antoniou, C., 2020. Shared autonomous vehicle services: A comprehensive review. Transportation Research Part C: Emerging Technologies 111, 255 - 293. URL: http://www.sciencedirect. com/science/article/pii/S0968090X19303493 doi https://doi.org/10.1016/j.trc.2019.12.008

Olia, A., Razavi, S., Abdulhai, B., Abdelgawad, H., 2018. Traffic capacity implications of automated vehicles mixed with regular vehicles. Journal of Intelligent Transportation Systems 22, 244 262. URL: https://doi.org/10.1080/15472450.2017.1404680 doi 10.1080/15472450.2017.1404680 arXiv:https://doi.org/10.1080/15472450.2017.1404680

Pinto, H.K., Hyland, M.F., Mahmassani, H.S., Omer Verbas, I., 2020. Joint design of multimodal transit networks and shared autonomous mobility fleets. Transportation Research Part C: Emerging Technologies 113 2 - 20. URL: http://www.sciencedirect.com/science/article/pii/S0968090X18317728. doi https: //doi.org/10.1016/j.trc.2019.06.010 23rd International Symposium on Transportation and Traffic Theory (ISTTT 23).

Ritzinger, U., Puchinger, J., Hartl, R.F., 2016. Dynamic programming based metaheuristics for the dial-a-ride problem. Annals of Operations Research 236, 341-358. URL: https://doi .org/10.1007/s10479-014-1605-7. doi 10. 
1007/s10479-014-1605-7

Ropke, S., Cordeau, J.F., 2006. Heuristic and exact algorithms for vehicle routing problems. Ph.D. thesis. University of Copenhagen. Branch-and-cut-and-price for the pickup and delivery problem with time windows.

Ropke, S., Cordeau, J.F., 2009. Branch and cut and price for the pickup and delivery problem with time windows. Transportation Science 43, 267-286. URL: https://doi.org/10.1287/trsc.1090.0272 doi $10.1287 /$ trsc. 1090.0272 arXiv:https://doi.org/10.1287/trsc.1090.0272

Rousseau, L.M., Gendreau, M., Feillet, D., 2007. Interior point stabilization for column generation. Operations Research Letters 35, 660 - 668. URL:/http://www.sciencedirect.com/science/article/pii/S0167637706001349 doi https://doi.org/10.1016/j.orl.2006.11.004

Rousseau, L.M., Gendreau, M., Pesant, G., Focacci, F., 2004. Solving vrptws with constraint programming based column generation. Annals of Operations Research 130, 199-216. URL: https://doi.org/10.1023/B: ANOR. 0000032576.73681 .29 doi $10.1023 /$ B:ANOR.0000032576.73681.29

Ruland, K., Rodin, E., 1997. The pickup and delivery problem: Faces and branch-and-cut algorithm. Computers \& Mathematics with Applications 33, 1 - 13. URL: http://www.sciencedirect.com/science/article/pii/ S0898122197000904 doi https://doi.org/10.1016/S0898-1221(97)00090-4

Salazar, M., Rossi, F., Schiffer, M., Onder, C.H., Pavone, M., 2018. On the interaction between autonomous mobilityon-demand and public transportation systems, in: 2018 21st International Conference on Intelligent Transportation Systems (ITSC), pp. 2262-2269. doi 10.1109/ITSC.2018.8569381

Santi, P., Resta, G., Szell, M., Sobolevsky, S., Strogatz, S.H., Ratti, C., 2014. Quantifying the benefits of vehicle pooling with shareability networks. Proceedings of the National Academy of Sciences 111 13290-13294. URL: https://www.pnas.org/content/111/37/13290 doi 10.1073/pnas.1403657111 arXiv:https://www.pnas.org/content/111/37/13290.full.pdf

Savelsbergh, M.W.P., 1985. Local search in routing problems with time windows. Annals of Operations Research 4, 285-305. URL:https://doi.org/10.1007/BF02022044 doi 10.1007/BF02022044

Soteropoulos, A., Berger, M., Ciari, F., 2019. Impacts of automated vehicles on travel behaviour and land use: an international review of modelling studies. Transport Reviews 39, 29-49. URL: https://doi.org/10.1080/01441647.2018.1523253 doi 10.1080/01441647.2018.1523253 arXiv:https://doi.org/10.1080/01441647.2018.1523253

Stocker, A., Shaheen, S., 2019. Shared automated vehicle (sav) pilots and automated vehicle policy in the u.s.: Current and future developments, in: Meyer, G., Beiker, S. (Eds.), Road Vehicle Automation 5, Springer International Publishing, Cham. pp. 131-147.

Taillard, É., Badeau, P., Gendreau, M., Guertin, F., Potvin, J.Y., 1997. A tabu search heuristic for the vehicle routing problem with soft time windows. Transportation Science 31, 170-186. URL: https://doi.org/10.1287/trsc . 31.2 .170 doi $10.1287 / \operatorname{trsc} .31 .2 .170$ arXiv:https://doi.org/10.1287/trsc.31.2.170

Talebpour, A., Mahmassani, H.S., 2016. Influence of connected and autonomous vehicles on traffic flow stability and throughput. Transportation Research Part C: Emerging Technologies 71, $143-163$. URL: http:// Www.sciencedirect.com/science/article/pii/S0968090X16301140 doi/https://doi.org/10.1016/ j.trc.2016.07.007

Tang, J., Kong, Y., Lau, H., Ip, A.W., 2010. A note on "efficient feasibility testing for dial-a-ride problems". Operations Research Letters 38, 405 - 407. URL: http://www.sciencedirect.com/science/article/pii/ S016763771000057X doi https://doi.org/10.1016/j.orl.2010.05.002

Thomas, M., Deepti, T., 2018. Reinventing carsharing as a modern and profitable service. The Intelligent Transportation Society of America 2018 Annual Meeting White Paper. URL: https://ridecell.com/wp-content/uploads/ White-Paper-Presentation_Reinventing-Carsharing-As-A-Modern-And-Profitable-Service.pdf

Tientrakool, P., Ho, Y., Maxemchuk, N.F., 2011. Highway capacity benefits from using vehicle-to-vehicle communication and sensors for collision avoidance, in: 2011 IEEE Vehicular Technology Conference (VTC Fall), pp. 1-5. doi 10.1109/VETECF. 2011.6093130

Wang, H., Cheu, R.L., Lee, D.H., 2014. Intelligent taxi dispatch system for advance reservations. Journal of Public Transportation 17, 115-128. URL: https://scholarcommons.usf.edu/jpt/vol17/iss3/8 doi http: //doi.org/10.5038/2375-0901.17.3.8

Zhang, W., Guhathakurta, S., 2017. Parking spaces in the age of shared autonomous vehicles: How much parking will we need and where? Transportation Research Record 2651, 80-91. URL: https://doi.org/10.3141/2651-09 doi 10.3141/2651-09 arXiv:https://doi.org/10.3141/2651-09

Zhang, W., Guhathakurta, S., Fang, J., Zhang, G., 2015. Exploring the impact of shared autonomous vehicles on urban parking demand: An agent-based simulation approach. Sustainable Cities and Society 19, 34 - 45. URL: http://www.sciencedirect.com/science/article/pii/S221067071530010X doi https: //doi.org/10.1016/j.scs.2015.07.006 


\section{Appendix A. The Pricing Subproblem PSP CTSPAV}

Construction of graphs for PSP CTSPAV. Let $v_{t}^{i}$ denote a virtual sink node for graph $\mathcal{G}_{i}^{+}$or $\mathcal{G}_{i}^{-}$. Since a mini route covers only inbound trips or outbound trips, $\mathcal{G}_{i}^{+}=\left(\mathcal{N}_{i}^{+}, \mathcal{A}_{i}^{+}\right)\left(\right.$resp. $\mathcal{G}_{i}^{-}=$ $\left(\mathcal{N}_{i}^{-}, \mathcal{A}_{i}^{-}\right)$) contains, in addition to $v_{t}^{i}$, only the nodes for inbound trips (resp. outbound trips), i.e., $\mathcal{N}_{i}^{+}=\mathcal{P}^{+} \cup \mathcal{D}^{+} \cup\left\{v_{t}^{i}\right\}$ (resp. $\mathcal{N}_{i}^{-}=\mathcal{P}^{-} \cup \mathcal{D}^{-} \cup\left\{v_{t}^{i}\right\}$ ). The set $\mathcal{A}_{i}^{+}$(resp. $\mathcal{A}_{i}^{-}$) then represents all feasible edges for $\mathcal{G}_{i}^{+}$(resp. $\mathcal{G}_{i}^{-}$), i.e., location pairs from $\mathcal{N}_{i}^{+}$(resp. $\mathcal{N}_{i}^{-}$) that satisfy a priori route feasibility constraints. Without loss of generality, the following elaborates further on how $\mathcal{G}_{i}^{+}$is constructed.

Construction of $\mathcal{G}_{i}^{+}=\left(\mathcal{N}_{i}^{+}, \mathcal{A}_{i}^{+}\right)$begins with the introduction of the set of nodes $\mathcal{N}_{i}^{+}=$ $\mathcal{P}^{+} \cup \mathcal{D}^{+} \cup\left\{v_{t}^{i}\right\}$ and a set of fully-connected edges $\mathcal{A}_{i}^{+}=\left\{(u, v): u, v \in \mathcal{N}_{i}^{+}, u \neq v\right\}$. A ride-duration limit $L_{u}$ is associated with each node $u \in \mathcal{P}^{+}$, a time window $\left[a_{u}, b_{u}\right]$ and service duration $s_{u}$ are associated with each node $u \in \mathcal{P}^{+} \cup \mathcal{D}^{+}$, and a travel time $\tau_{(u, v)}$ and a reduced cost $\bar{c}_{(u, v)}$ are associated with each edge $(u, v) \in \mathcal{A}_{i}^{+}$. As the goal is to find a feasible mini route from $i$ to $v_{t}^{i}$ with minimum reduced cost, $\bar{c}_{(u, v)}$ is defined as follows so that the total cost of any path from $i$ to $v_{t}^{i}$ is equivalent to that defined in 24].

$$
\bar{c}_{(u, v)}= \begin{cases}-\pi_{u}-\mu_{(u, v)} & \forall(u, v) \in \mathcal{A}_{i}^{+} \backslash \delta^{-}\left(v_{t}^{i}\right): u \in \mathcal{P}^{+} \\ -\mu_{(u, v)} & \forall(u, v) \in \mathcal{A}_{i}^{+} \backslash \delta^{-}\left(v_{t}^{i}\right): u \in \mathcal{D}^{+} \\ 0 & \forall(u, v) \in \delta^{-}\left(v_{t}^{i}\right)\end{cases}
$$

Edges from $\mathcal{A}_{i}^{+}$that cannot belong to any feasible mini route are then identified by pre-processing time-window, pairing, precedence, and ride-duration limit constraints. Prior to this pre-processing step, knowledge of $i$ being the source of the path sought from $\mathcal{G}_{i}^{+}$allows the time windows of all nodes $u \in \mathcal{P}^{+} \cup \mathcal{D}^{+}$to be tightened, by sequentially increasing their lower bounds using the following rules proposed by Dumas et al. (1991):

- $a_{u}=\max \left\{a_{u}, a_{i}+s_{i}+\tau_{(i, u)}\right\}, \forall u \in \mathcal{P}^{+} \backslash\{i\}$

- $a_{n+u}=\max \left\{a_{n+u}, a_{u}+s_{u}+\tau_{(u, n+u)}\right\}, \forall u \in \mathcal{P}^{+} \backslash\{i\}$

The following sets of infeasible edges are then identified and consequently removed from $\mathcal{A}_{i}^{+}$:

(a) Direct trips to source $i$ and from sink $v_{t}^{i}$ : $\delta^{-}(i) \cup \delta^{+}\left(v_{t}^{i}\right)$

(b) Precedence of pickup and drop-off nodes:

- $\left\{(i, v): v \in \mathcal{D}^{+} \backslash\{n+i\}\right\}$

- $\left\{\left(u, v_{t}^{i}\right): u \in \mathcal{P}^{+}\right\}$

- $\left\{(u, v): u \in \mathcal{D}^{+} \wedge v \in \mathcal{P}^{+}\right\}$

(c) Time windows along each edge: $\left\{(u, v):(u, v) \in \mathcal{A}_{i}^{+} \backslash \delta^{-}\left(v_{t}^{i}\right) \wedge a_{u}+s_{u}+\tau_{(u, v)}>b_{v}\right\}$

(d) Ride-duration limit of each commuter: $\left\{(u, v),(v, n+u): u \in \mathcal{P}^{+} \wedge v \in \mathcal{P}^{+} \cup \mathcal{D}^{+} \wedge u \neq\right.$ $\left.v \wedge \tau_{(u, v)}+s_{v}+\tau_{(v, n+u)}>L_{u}\right\}$

(e) Time windows and ride-duration limits of pairs of trips:

- $\left\{(u, n+v): u, v \in \mathcal{P}^{+} \wedge u \neq v \wedge \neg\right.$ feasible $\left.(v \rightarrow u \rightarrow n+v \rightarrow n+u)\right\}$

- $\left\{(n+u, v): u, v \in \mathcal{P}^{+} \wedge u \neq v \wedge \neg\right.$ feasible $\left.(u \rightarrow n+u \rightarrow v \rightarrow n+v)\right\}$

- $\left\{(u, v): u, v \in \mathcal{P}^{+} \wedge u \neq v \wedge \neg\right.$ feasible $(u \rightarrow v \rightarrow n+u \rightarrow n+v) \wedge \neg$ feasible $(u \rightarrow v \rightarrow$ $n+v \rightarrow n+u)\}$ 


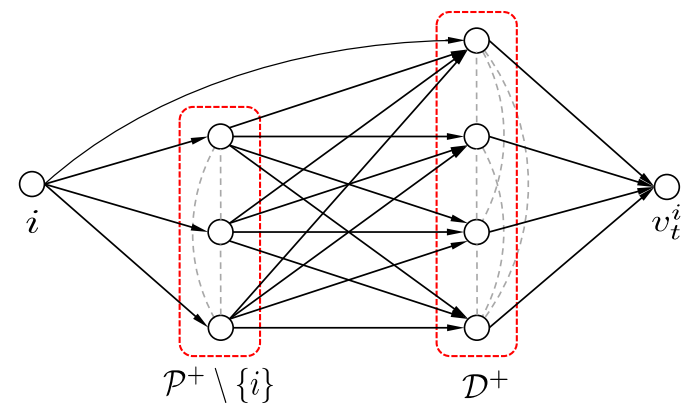

Figure A.32: Graph $\mathcal{G}_{i}^{+}$(Each Dotted Line Represents a Pair of Bidirectional Edges).

- $\left\{(n+u, n+v): u, v \in \mathcal{P}^{+} \wedge u \neq v \wedge \neg\right.$ feasible $(u \rightarrow v \rightarrow n+u \rightarrow n+v) \wedge \neg$ feasible $(v \rightarrow$ $u \rightarrow n+u \rightarrow n+v)\}$

An example of graph $\mathcal{G}_{i}^{+}$that results from the removal of the infeasible edges is shown in Figure A.32

Search for path with minimum reduced cost. The final step in PSP $\mathrm{CTSPAV}_{\text {in }}$ is to find a path from $i$ to $v_{t}^{i}$ from each graph $\mathcal{G}_{i}^{+}: i \in \mathcal{P}^{+}$and $\mathcal{G}_{i}^{-}: i \in \mathcal{P}^{-}$. On top of having the minimum cost, the path must also represent a feasible mini route. Notice that, as shown in Figure A.32, construction of the graphs eliminates all edges $\{(u, v): u \in \mathcal{D} \wedge v \in \mathcal{P}\}$. Therefore, any path from $i$ to $v_{t}^{i}$ is guaranteed to begin with a pickup phase that only visits nodes in $\mathcal{P}$ and end with a drop-off phase that only visits nodes in $\mathcal{D}$. In other words, the graphs ensure the precedence feasibility constraint, which requires all pickup nodes of a mini route to precede all of its drop-off nodes, is satisfied by construction. All that remains to guarantee the feasibility of any path found is to ensure that the path:

1. Visits each node within its specified time window,

2. Visits the corresponding drop-off nodes of every pickup node visited,

3. Satisfies the ride-duration limit of each rider served,

4. Respects the vehicle capacity, and

5. Visits each node at most once (i.e., it is elementary).

The problem of finding a feasible, least-cost path from $i$ to $v_{t}^{i}$ is essentially an Elementary Shortest Path Problem with Resource Constraints (ESPPRC) (Irnich and Desaulniers, 2005), whereby the remaining route-feasibility constraints can be modeled with resource constraints. In fact, this ESPPRC is identical to that in the column-generation pricing problem of the DARP considered in Gschwind and Irnich (2015). They proposed a dynamic-programming, label-setting algorithm that uses strong label dominance rules to find the optimal solution to the problem.

While their algorithm can also be used to effectively solve this work's ESPPRC, the algorithm has a pre-requisite; it requires that the edge reduced costs $\bar{c}_{(u, v)}$ satisfy the delivery triangle inequality (DTI). The DTI, introduced by Ropke and Cordeau (2009), requires that the reduced costs fulfill $\bar{c}_{(u, v)} \leq \bar{c}_{(u, w)}+\bar{c}_{(w, v)}$ for all edges $(u, v),(u, w),(w, v) \in\left\{\mathcal{A}_{i}^{+}: i \in \mathcal{P}^{+}\right\} \cup\left\{\mathcal{A}_{i}^{-}: i \in \mathcal{P}^{-}\right\}$ and $w \in \mathcal{D}$. Unfortunately, the reduced costs in this problem do not satisfy the DTI. A costmatrix transformation, also proposed by Ropke and Cordeau (2009), is therefore first applied to the reduced costs to transform them into an equivalent set of costs that does satisfy the DTI, 
after which the label-setting algorithm of Gschwind and Irnich (2015) is applied on each graph $\mathcal{G}_{i}^{+}: i \in \mathcal{P}^{+}$and $\mathcal{G}_{i}^{-}: i \in \mathcal{P}^{-}$to find the feasible, least-cost paths from $i$ to $v_{t}^{i}$. Finally, note that enforcement of pairing and precedence resource constraints of the label-setting algorithm on the origin and destination node pairs of the graphs are sufficient to ensure elementarity of the paths produced, as the graphs lack edges $\{(u, v): u \in \mathcal{D} \wedge v \in \mathcal{P}\}$ which are necessary to produce cycles in the presence of the pairing and precedence constraints. Therefore, additional resource constraints dedicated specifically to ensure elementarity of the paths are not necessary for PSP $\mathrm{CTSPAV}_{\text {. }}$

\section{Appendix B. Optimality Gaps and Computation Times}

The optimality gaps and computation times of all experiments for the CTSPAV procedure are summarized in Tables B.2 and B.3. In Table B.2, which summarizes the results of problem instances for the lexicographic objective, the first two columns specify the location of the clusters and the configuration of their depots. The following two columns list average values of the absolute gap for the vehicle count. The absolute gap is calculated by taking the vehicle count results of the MIP and subtracting from it its lower bound. The first gap uses results the from $\mathrm{RMP}_{\mathrm{CTSPAV}}$. Letting $Y_{e}^{*}$ be the value of $Y_{e}$ from RMP $\mathrm{RTSPAV}_{\text {at }}$ anvergence, the primal lower bound to the vehicle count is given by $\left\lceil\sum_{e \in \delta\left(v_{s}\right)} Y_{e}^{*}\right\rceil$. The second gap uses the primary objective results of $\mathrm{RMP}_{\mathrm{DARP}}$, particularly $\left\lceil z_{\mathrm{LB}}^{\prime}\right\rceil$ as the vehicle count lower bound. It was found to consistently provide a stronger lower bound than the former, and therefore it is included here to provide an additional perspective. The next column shows the average optimality gap, which is given by $\left(z_{\text {MIP }}-z^{*}\right) / z_{\text {MIP }}$ for each instance, where $z_{\text {MIP }}$ denotes the MIP's final objective value, and $z^{*}$, the objective value of RMP $\mathrm{CTSPAV}_{\text {at }}$ at convergence, provides a primal lower bound for $z_{\text {MIP. }}$. However, for a few problem instances representing clusters outside city limits, the column-generation phase did not converge within the time limit. For these instances, the dual lower bound $z_{\mathrm{LB}}$ is used in place of $z^{*}$ when calculating the optimality gap, and the vehicle count lower bound from the CTSPAV procedure is obtained by considering only the fixed cost contributions to $z_{\mathrm{LB}}$. All uncertainties are represented by the standard error of the mean.

While the average optimality gap for the lexicographic objective is relatively high at approximately $70 \%$ (resp. 76\%) for clusters inside the city (resp. outside the city), the vehicle count gap, which depicts the absolute gap of the primary objective, paints a different picture, averaging at 2.5 and 7.0 vehicles for clusters inside and outside city limits respectively. Moreover, when the lower bounds produced by the DARP procedure are used, the vehicle count gap averages at even lower values of 1.1 and 4.2 vehicles for clusters inside and outside city limits respectively. These lower values highlight a key strength of the DARP procedure, it consistently produces stronger lower bounds for the primary objective. When these stronger lower bounds are used, some problem instances produced an absolute vehicle count gap of zero, indicating that their primary objective results are optimal.

The next column shows the average number of mini routes, i.e. columns, generated in the column-generation phase, while the following two columns summarize the time spent in this phase. The latter of the two summarizes the fraction of problem instances in which the columngeneration phase met or exceeded the 1 hour time limit (i.e., the fraction of instances whereby the column-generation phase did not converge within the time limit), while the former shows the average wall time spent on column generation for problem instances that did not exceed the time limit. It can be seen that on average, the time spent on column generation is significantly higher on clusters outside city limits. On top of that, more than $30 \%$ of the instances did not achieve 
Table B.2: Optimality Gaps and Computation Times of the CTSPAV Procedure with the Lexicographic Objective

\begin{tabular}{ccccccccc}
\hline \multirow{2}{*}{$\begin{array}{c}\text { Cluster } \\
\text { location }\end{array}$} & $\begin{array}{c}\text { Depot } \\
\text { config. }\end{array}$ & $\begin{array}{c}\text { Average } \\
\text { vehicle } \\
\text { count gap }\end{array}$ & $\begin{array}{c}\text { Average } \\
\text { vehicle } \\
\text { count gap } \\
\text { (DARP LB) }\end{array}$ & $\begin{array}{c}\text { Average } \\
\text { optimality } \\
\text { gap }(\%)\end{array}$ & $\begin{array}{c}\text { Average } \\
\text { mini route } \\
\text { count }\end{array}$ & $\begin{array}{c}\text { Average } \\
\text { colgen } \\
\text { time (s) }\end{array}$ & $\begin{array}{c}\% \geq \\
\text { colgen } \\
\text { time } \\
\text { limit }\end{array}$ & $\begin{array}{c}\% \geq \\
\text { MIP } \\
\text { time } \\
\text { limit }\end{array}$ \\
\hline $\begin{array}{c}\text { Inside } \\
\text { city }\end{array}$ & Central & $2.55 \pm 0.14$ & $1.09 \pm 0.15$ & $70.5 \pm 1.4$ & $5394 \pm 741$ & $86 \pm 28$ & 0.0 & 100.0 \\
\cline { 2 - 9 }$y$ & Local & $2.50 \pm 0.16$ & $1.09 \pm 0.16$ & $69.8 \pm 1.7$ & $5699 \pm 893$ & $161 \pm 84$ & 0.0 & 100.0 \\
\hline $\begin{array}{c}\text { Outside } \\
\text { city }\end{array}$ & Central & $7.07 \pm 0.45$ & $4.19 \pm 0.28$ & $75.5 \pm 2.1$ & $19949 \pm 1021$ & $926 \pm 141$ & 32.4 & 100.0 \\
\cline { 2 - 9 }$y$ & Local & $7.03 \pm 0.47$ & $4.12 \pm 0.30$ & $75.5 \pm 2.2$ & $19631 \pm 974$ & $922 \pm 133$ & 36.8 & 100.0 \\
\hline
\end{tabular}

Table B.3: Optimality Gaps and Computation Times of the CTSPAV Procedure with the Distance Minimization Objective

\begin{tabular}{ccccccc}
\hline $\begin{array}{c}\text { Cluster } \\
\text { location }\end{array}$ & $\begin{array}{c}\text { Depot } \\
\text { config. }\end{array}$ & $\begin{array}{c}\text { Average } \\
\text { optimality } \\
\text { gap }(\%)\end{array}$ & $\begin{array}{c}\text { Average } \\
\text { mini route } \\
\text { count }\end{array}$ & $\begin{array}{c}\text { Average } \\
\text { colgen } \\
\text { time (s) }\end{array}$ & $\begin{array}{c}\% \geq \text { colgen } \\
\text { time limit }\end{array}$ & $\begin{array}{c}\% \geq \text { MIP } \\
\text { time limit }\end{array}$ \\
\hline $\begin{array}{c}\text { Inside } \\
\text { city }\end{array}$ & Central & $1.75 \pm 0.17$ & $5396 \pm 752$ & $73 \pm 22$ & 0.0 & 36.4 \\
\cline { 2 - 7 } & Local & $1.35 \pm 0.17$ & $5453 \pm 803$ & $105 \pm 37$ & 0.0 & 13.6 \\
\hline $\begin{array}{c}\text { Outside } \\
\text { city }\end{array}$ & Central & $3.05 \pm 0.48$ & $20533 \pm 1147$ & $960 \pm 112$ & 8.8 & 82.4 \\
\cline { 2 - 7 } & Local & $4.75 \pm 1.04$ & $21114 \pm 1140$ & $1126 \pm 129$ & 19.1 & 73.5 \\
\hline
\end{tabular}

convergence within the time limit. This can be attributed to the significantly larger number of columns generated from these clusters. Finally, the last column shows the fraction of problem instances in which the MIP solving phase met or exceeded its 1 hour time budget. For the lexicographic objective, the time limit was exceeded on all problem instances.

Table B.3 summarizes the results of problem instances for the distance-minimization objective. It displays the same information as Table B.2. except that it does not list the average vehicle count gaps. This is due to the lower bound of the vehicle count not being available from the objective. It can be seen that the optimality gap for the distance-minimization objective is excellent, being less than $2 \%$ on average for cases inside the city and less than $5 \%$ on average for cases outside. Similar to instances for the lexicographic objective, the average number of columns generated from clusters outside city limits is significantly larger than that from clusters inside, which consequently results in the significantly longer average time spent on the columngeneration phase. Finally, the percentage of problem instances that exceed the time limits of the column-generation and MIP phases is fewer than that for the lexicographic objective in every case, and this can be attributed to the relatively stronger primal lower bound provided by the LP relaxation of $\mathrm{MP}_{\mathrm{CTSPAV}}$ when the distance-minimization objective is utilized.

Table B.4 summarizes the average optimality gaps and computations times of the same set of problem instances for the DARP procedure with the lexicographic objective. Once again, all uncertainties are represented by the standard error of the mean. Its first two columns specify the cluster location and depot configuration of the instances. The next lists the average number of 
Table B.4: Optimality Gaps and Computation Times of the DARP Procedure with the Lexicographic Objective

\begin{tabular}{ccccccccc}
\hline \multirow{2}{*}{$\begin{array}{c}\text { Cluster } \\
\text { location }\end{array}$} & $\begin{array}{c}\text { Depot } \\
\text { config. }\end{array}$ & $\begin{array}{c}\text { Average } \\
\text { column } \\
\text { count }\end{array}$ & $\begin{array}{c}\text { Average } \\
\text { primary } \\
\text { absolute } \\
\text { gap }\end{array}$ & $\begin{array}{c}\text { Average } \\
\text { primary } \\
\text { optimality } \\
\text { gap }(\%)\end{array}$ & $\begin{array}{c}\% \geq \\
\text { primary } \\
\text { colgen } \\
\text { time limit }\end{array}$ & $\begin{array}{c}\% \geq \\
\text { colgen } \\
\text { time } \\
\text { limit }\end{array}$ & $\begin{array}{c}\text { MIP time } \\
\text { primary } \\
\text { limit }\end{array}$ & $\begin{array}{c}\text { MIP } \\
\text { time } \\
\text { limit }\end{array}$ \\
\hline $\begin{array}{c}\text { Inside } \\
\text { city }\end{array}$ & Central & $34107 \pm 5602$ & $2.68 \pm 0.14$ & $47.9 \pm 2.1$ & 68.2 & 100.0 & 18.2 & 36.4 \\
\cline { 2 - 9 } & Local & $33911 \pm 5554$ & $2.55 \pm 0.16$ & $49.0 \pm 2.7$ & 68.2 & 100.0 & 9.1 & 27.3 \\
\hline \multirow{2}{*}{$\begin{array}{c}\text { Outside } \\
\text { city }\end{array}$} & Central & $6801 \pm 907$ & $4.81 \pm 0.23$ & $48.2 \pm 1.5$ & 95.6 & 98.5 & 14.7 & 25.0 \\
\cline { 2 - 9 } & Local & $6698 \pm 870$ & $4.79 \pm 0.25$ & $47.6 \pm 1.6$ & 95.6 & 98.5 & 14.7 & 19.1 \\
\hline
\end{tabular}

columns generated, followed by two columns which show the average absolute gap and average optimality gap of the primary objective value. Letting $z_{\mathrm{MIP}}^{\prime}$ denote the final primary objective value of the MIP and recalling that $z_{\mathrm{LB}}^{\prime}$ denotes its dual lower bound, the absolute gap of an instance given by $z_{\mathrm{MIP}}^{\prime}-\left\lceil z_{\mathrm{LB}}^{\prime}\right\rceil$ while its optimality gap is given by $\left(z_{\mathrm{MIP}}^{\prime}-\left\lceil z_{\mathrm{LB}}^{\prime}\right\rceil\right) / z_{\mathrm{MIP}}^{\prime}$. The average optimality gaps are relatively high at approximately $48 \%$. However, the absolute gaps reveal a different story, whereby they average at only 2.6 and 4.8 vehicles inside and outside city limits respectively. These values, however, are higher than those of the CTSPAV procedure which utilize the same lower bounds $\left(\left\lceil z_{\mathrm{LB}}^{\prime}\right\rceil\right)$.

The next column specifies the fraction of problem instances whereby the column-generation phase for the primary objective did not converge within the time limit, followed by one which specifies the fraction of instances whereby the column-generation phase for both objectives did not converge. When only the primary objective is considered, column-generation for more than two-thirds of the instances inside the city and almost all instances outside the city did not converge. On top of that, out of the 180 problem instances in total, only two had their columngeneration phases for both objectives converge within the allocated time limit, even after more time has been allocated for this phase (recall 1.5 hours is allocated for this phase of the DARP procedure as opposed to only 1 hour for the CTSPAV procedure). This highlights the harder nature of PSP $\mathrm{DARP}_{\text {P }}$ which searches for longer $\mathrm{AV}$ routes (as opposed to PSP $\mathrm{CTSPAV}_{\text {which only }}$ searches for mini routes). Nevertheless, regardless of the column-generation phase not converging for most instances, the DARP procedure was still able to consistently produce stronger lower bounds for the primary objective.

The next column shows the fraction of problem instances in which the MIP for the primary objective exceeded the time limit, while the last shows the fraction of instances in which the MIP for both objectives exceeded the time limit. Both columns show that the MIP can be solved within the time limit for a majority of the problem instances, which is in stark contrast to the MIP of the CTSPAV procedure, whereby its longer time limit is exceeded for all problem instances. This difference can be attributed to the relatively easier MP $\mathrm{DARP}_{\mathrm{DA}}$ which just solves a set-covering problem (instead of the $\mathrm{MP}_{\mathrm{CTSPAV}}$ which solves a route-scheduling problem) and the stronger primal lower bound provided by its linear relaxation.

Table B.5 summarizes the average optimality gaps and computations times for the DARP procedure with the distance-minimization objective. Similar to Table B.4 the first three columns specify cluster locations, depot configurations, and the average number of columns generated. The following two show average optimality gaps of the final MIP results. The first uses the 
Table B.5: Optimality Gaps and Computation Times of the DARP Procedure with the Distance Minimization Objective

\begin{tabular}{ccccccc}
\hline \multirow{2}{*}{$\begin{array}{c}\text { Cluster } \\
\text { location }\end{array}$} & $\begin{array}{c}\text { Depot } \\
\text { config. }\end{array}$ & $\begin{array}{c}\text { Average } \\
\text { column } \\
\text { count }\end{array}$ & $\begin{array}{c}\text { Average } \\
\text { optimality } \\
\text { gap (\%) }\end{array}$ & $\begin{array}{c}\text { Average } \\
\text { optimality } \\
\text { gap (\%) } \\
\text { (CTSPAV LB) }\end{array}$ & $\begin{array}{c}\% \geq \text { colgen } \\
\text { time limit }\end{array}$ & $\begin{array}{c}\% \geq \text { MIP } \\
\text { time limit }\end{array}$ \\
\hline $\begin{array}{c}\text { Inside } \\
\text { city }\end{array}$ & Central & $34286 \pm 5323$ & $98.0 \pm 2.0$ & $28.5 \pm 1.7$ & 100.0 & 77.3 \\
\cline { 2 - 7 } & Local & $30712 \pm 4640$ & $95.2 \pm 3.8$ & $36.4 \pm 3.1$ & 100.0 & 77.3 \\
\hline $\begin{array}{c}\text { Outside } \\
\text { city }\end{array}$ & Central & $6154 \pm 902$ & $94.8 \pm 2.6$ & $28.4 \pm 1.6$ & 96.7 & 36.7 \\
\cline { 2 - 7 } & Local & $5816 \pm 838$ & $95.5 \pm 2.6$ & $44.5 \pm 1.8$ & 96.9 & 37.5 \\
\hline
\end{tabular}

dual lower bound, $\left\lceil z_{\mathrm{LB}}\right\rceil$, in the gap calculation, whereas the second uses the primal lower bound produced by the CTSPAV procedure, $z^{*}$. For the distance-minimization objective, the CTSPAV procedure consistently produces stronger lower bounds, therefore optimality gaps calculated using its lower bound are also smaller. The dual lower bound $\left\lceil z_{\mathrm{LB}}\right\rceil$ is weaker in this case because the column-generation phase could not be completed within the time limit for almost all problem instances. This is further highlighted by the next column which shows the fraction of problems in which the column-generation phase could not be completed. Out of the 180 problem instances in total, only four managed to achieve convergence. For these four instances the strength of $\left\lceil z_{\mathrm{LB}}\right\rceil$ is comparable to $z^{*}$ from the CTSPAV procedure. Finally, the last column shows the fraction of problem instances in which the MIP exceeded its time limit. A larger fraction of instances inside the city exceeded the time limit due to their relatively larger column counts. 